\title{
The Evolution of Actuality to Probability
}

If, AS Heisenberg SUGGeSTED, potentia indeed constitute an ontologically fundamental species of reality, then the mathematical representation of the quantum mechanical evolution of the matrix of potentia to the matrix of probabilities - that is, the valuation of potentia as probabilities between zero and one-ought to be heuristically useful in understanding the ontological implications of this evolution. The mathematical representation should prove helpful in visualizing not only the process described by quantum mechanics but also, as we shall see in the following chapters, the process described by Whitehead. The basic formalism introduced and explored in this chapter is intended to be comprehensible to readers unfamiliar with mathematics. Some familiarity with the Pythagorean theorem and the addition of vectors will be useful, but not necessary.

\section{The Formal Description of Quantum Mechanical State Evolution}

As mentioned earlier, we refer to the "state" of a system as a maximal specification of the facts or actualities comprised by the system. (These facts/actualities are typically referred to by physicists as "observables" or "collective observables," even though most are, for all practical purposes, unobservable; for this reason, we will use the terms "facts" and "actualities" instead.) For the sake of simplicity, let us consider an idealized system consisting of nothing other than an old-fashioned traffic signal-the type with two lights, red and green. Let us further suppose that the signal always functions normally, such that neither of the lights is burned out and that both will never be illuminated simultaneously. And finally, let us ignore everything other than the status of the two lights - the wiring, the 
casing, and so on-such that the state of our idealized traffic-light system entails but one fact: The status of the signal, which is either green or red.

The state of any system in quantum mechanics is represented by a vector of unit length in Hilbert space-an abstract linear vector space that, in our idealized example, can be depicted via a simple $x-y$ Cartesian coordinate system. The benefit of using Hilbert spaces in quantum mechanics is that these spaces are capable of representing, in a mathematically useful way, potentia as well as actualities and their relationship in a given system. This representation is based on two simple principles:

(i) Every physical system can be represented by a unique Hilbert space $\mathscr{H}_{s}$, and the state $S$ of a given physical system (again, "state" being the maximal specification of the facts/actualities/observables associated with the system) can be represented by a single vector $|u\rangle$ of unit length in the system's Hilbert space.

(ii) In a measurement interaction involving the system, there is a oneto-one correspondence between the number of probability-valuated outcome system states and the number of dimensions comprised by the Hilbert space.

In the case of our highly idealized traffic-light system, which has two potential states $S$, then, the associated Hilbert space has only two dimensions: One represents " $S=$ green," and the other " $S=$ red." This particular two-dimensional Hilbert space is therefore easily represented by simple $x-y$ coordinate axes, where a vector of unit length $\left|u_{\text {green }}\right\rangle$ along the $x$-axis represents " $S=$ green" and a vector of unit length $\left|u_{\text {red }}\right\rangle$ along the $y$-axis represents " $\mathrm{S}=$ red."

As regards our idealized system, one can at this point see the logical need for Principle 2: It guarantees, in satisfaction of the logical principles of non-contradiction and the excluded middle, that there exist some potential states that are mutually exclusive and exhaustive, as represented by the mutual orthogonality of the $x$ and $y$ dimensions (where "mutually orthogonal vectors" are vectors at right angles to one another). To generalize this somewhat, we can say that in a Hilbert space of $n$ dimensions, there are only $n$ mutually orthogonal vectors, representing only $n$ mutually exclusive states. If, for example, we were dealing with a more modern traffic-signal system with a green, a red, and a yellow light, our Hilbert space would require three dimensions, represented by $x, y$, and $z$ coordinate axes. It 


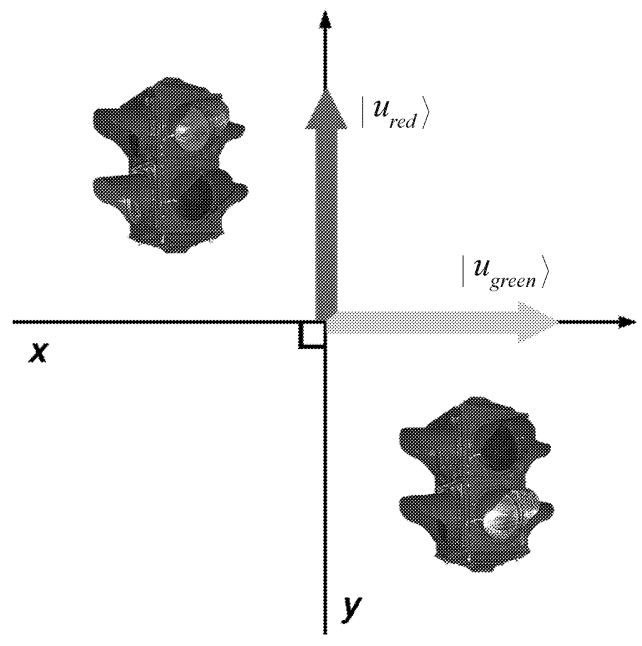

Figure 3.1 The two potential states $S$ of a traffic signal represented by orthogonal vectors of unit length in a Hilbert space of two dimensions. The vector $\left|u_{\text {green }}\right\rangle$ along the $x$-axis represents "S = green," and the vector $\left|u_{\text {red }}\right\rangle$ along the $y$-axis represents "S $=$ red."

is only by means of this third dimension that we are guaranteed three mutually exclusive and exhaustive states: $S=$ green, $S=$ yellow, or $S=$ red, represented by the mutual orthogonality of the vectors along the $x, y$, and $z$ axes. An added benefit of mutually orthogonal vectors representing mutually exclusive and exhaustive states is that such vectors can be grouped so that, as regards a modern trafficsignal system, we can specify the three possible states thus: $S$ is either green, represented by $\left|u_{\text {green }}\right\rangle$, or $S$ is not green, represented by the plane formed by $\left|u_{\text {red }}\right\rangle+\left|u_{\text {yellow }}\right\rangle ; S$ is either red $\left|u_{\text {red }}\right\rangle$ or not red $\left|u_{\text {green }}\right\rangle$ $+\left|u_{\text {yellow }}\right\rangle ; S$ is either yellow $\left|u_{\text {yellow }}\right\rangle$ or not yellow $\left|u_{\text {green }}\right\rangle+\left|u_{\text {red }}\right\rangle$.

Such groupings are referred to as "subspaces" of the Hilbert space, and their usefulness becomes readily apparent when considering nonidealized systems with manifold—even innumerable-potential states, each with a multiplicity of associated facts/actualities/observables. Consider, for example, the state of "System You" as you read this chapter. As is the case for most physical systems, a maximal specification of the facts/actualities/observables associated with you is far too unwieldy to calculate, so let us focus, as is typically done in quantum mechanics, on just one fact/actuality/observable: the location of System You in the universe. We first represent the state of 
System You via a vector of unit length somewhere in a Hilbert space of a certain number of dimensions-as many dimensions as there are places in the universe where you might be located. This is, of course, a practically infinite number of dimensions, regardless of our choice of location units-impossible to graph as we did with our idealized two- and three-dimensional examples. But conceptually, the principles are the same. We can say, then, that all of the practically infinite number of mutually orthogonal vectors of unit length in our Hilbert space represent potential, mutually exclusive and exhaustive states of System You. A certain number of these states include, among other facts/actualities/observables, locations of System You that are in the Amazon River. Again, the mutual orthogonality of the vectors provides for mutually exclusive potential states that, as regards location, prevent you from being two places at once. It is convenient to group all of these mutually orthogonal "Amazon River vectors" into a subspace $\mathscr{E}_{\text {amazon }}$.

Each vector represents a maximal specification of System You, including many facts/actualities/observables other than location; and whatever we might say about these other facts/actualities/observables, so long as a given vector belongs to the subspace $\mathscr{E}_{\text {amazon }}$, the location of System You is indeed in the Amazon. One vector in this subspace, $\left|u_{\text {hot }}\right\rangle$, for example, might represent a potential state where you are wearing a T-shirt in the Amazon, and another vector in this subspace, $\left|u_{\text {cold }}\right\rangle$, might represent a potential state where you are wearing a winter coat in the Amazon. These differences and a myriad of others aside, all vectors in the subspace $\mathscr{E}_{\text {amazon }}$ represent alternative states that share a single defining characteristic: the fact/actuality/observable "location" = "the Amazon River." The state of System You as regards location, then, can be expressed thus: you are either somewhere in the Amazon, or you are not. If the former, then your state is represented by some vector belonging to $\mathscr{E}_{\text {amazon; }}$ if the latter, then your state is represented by some vector outside of $\mathscr{E}_{\text {amazon }}$, which is itself a subspace, $\mathscr{E}_{\text {amazon }}{ }^{\text {}}$ consisting of all vectors orthogonal to those in $\mathscr{E}_{\text {amazon }}$.

In this way-unlike the classical mechanical conception of measurement as a qualification along a fluid continuum of qualities (consider the image of a "fluidly" sweeping second hand on an electric analog clock face, for example)-the conception of measure- 
ment in quantum mechanics entails the discrete specification of the truth or falsity of a particular fact/actuality/observable in a potential system state. A digital clock either reads "12:23 P.M." or it does not. A laser's wavelength is $650 \mathrm{~nm}$ when measured, or it is not. If the former, the vector representing the laser's state belongs to $\mathscr{E}_{650 \mathrm{~mm}}$ and if the latter, it belongs to $\mathscr{E}_{650 \mathrm{~mm}}{ }^{\mathrm{L}}$. If a traffic light is green, its state vector belongs to $\mathscr{E}_{\text {green }}$, and if it is not green, its state vector belongs to $\mathscr{E}_{\text {green }}{ }^{\perp}$.

This conception of measurement via explicit contrast-the discrete specification of the state of a system in terms of the truth or falsity (that is, the actuality) of a given potential fact associated with a potential state - and the mathematical representation of such contrasts via vectors in Hilbert space, provides not only a coherent framework for performing the relevant calculations of quantum mechanics, but also a coherent framework for exploring the relationship between potentiality and actuality in quantum mechanics, and the conceptually innovative ontological implications of this relationship.

The first step of this exploration begins with the following (and for many, infamous) quantum mechanical innovation, already introduced with a previous reference to Schrödinger's cat: There are vectors of unit length-that is, vectors that represent real states of a physical system - that belong neither to $\mathscr{E}$ nor to $\mathscr{E}^{\perp}$ for a given fact. There are, in other words, real physical states wherein the actuality of a given potential fact is objectively indefinite. Let us return to our old-fashioned, green-red traffic-light system, where a unit-length vector $\left|u_{\text {green }}\right\rangle$ along the $x$-axis represents the state $S=$ green, and a unit-length vector $\left|u_{\text {red }}\right\rangle$ along the $y$-axis represents $S=$ red. What of the unit-length vector $|\psi\rangle$, which lies neither on the $x$ nor $y$ axis? Since it is a vector of unit length, it represents a real potential physical state according to the quantum theory; but it represents a physical state wherein the traffic signal is neither green nor red-or similarly, a state wherein Schrödinger's cat is neither alive nor dead. Quantum mechanics describes the evolution of such "pure states" $|\psi\rangle$, whose potential facts are entangled in a superposition, into a finite set of mutually exclusive and exhaustive states (represented by a set of mutually orthogonal vectors). Each of these potential outcome states is assigned a probability, and all of the alternative outcome states together are referred to collectively as a "mixed state," so named because unlike a pure state $|\psi\rangle$, the mixed state comprises 
a mixture of valuated, mutually exclusive probable outcome states. In the case of our idealized, old-fashioned, red-green traffic-signal example, the pure state $|\psi\rangle$ evolves to become an eigenstate (one of the component states of the mixed state) where the signal is definitely either green $\left|u_{\text {green }}\right\rangle$ or red $\left|u_{\text {red }}\right\rangle$ upon observation (measurement). The state begins as the pure state, $|\psi\rangle$, where the two possible outcome states are correlated, or entangled. This is expressed as the sum of the associated vectors: $|\psi\rangle=\left|u_{\text {green }}\right\rangle+\left|u_{\text {red }}\right\rangle$. The vector $\left|u_{\text {green }}\right\rangle$ is added to $\left|u_{\text {red }}\right\rangle$ by simply transporting the tail of $\left|u_{\text {green }}\right\rangle$ to the head of $\left|u_{\text {red }}\right\rangle$ and then drawing a new vector $\left.|\psi\rangle\right\rangle$ from the origin to the transported head of $\left|u_{\text {green }}\right\rangle$.

It is thus easy to see why the pure state vector $|\psi\rangle$ can be expressed as the sum of two eigenvectors $\left|u_{\text {green }}\right\rangle$ or $\left|u_{\text {red }}\right\rangle$. The probability assigned to each eigenstate of the mixture is indicated by adding a complex coefficient $\alpha$ and $\beta$ to $\left|u_{\text {green }}\right\rangle$ and $\left|u_{\text {red }}\right\rangle$ respectively, which indicates the vector's length:

$$
|\psi\rangle=\alpha\left|u_{\text {green }}\right\rangle+\beta\left|u_{\text {red }}\right\rangle
$$

where $|\alpha|^{2}+|\beta|^{2}=1$.

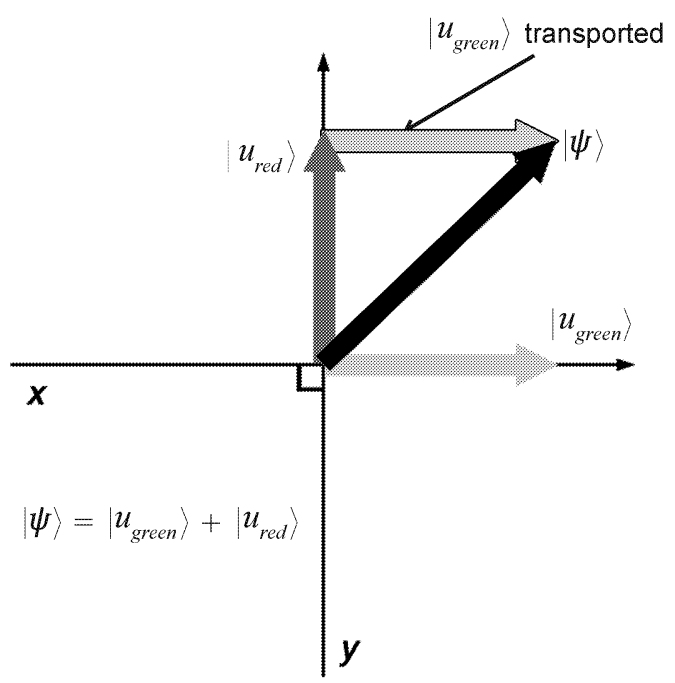

FIgURE $3.2|\psi\rangle$ is a vector of unit length, and therefore a real, but objectively indefinite state that is neither $\left|u_{\text {green }}\right\rangle$ nor $\left|u_{\text {red }}\right\rangle$ but is expressible as a sum of these two vectors. $\left|u_{\text {green }}\right\rangle$ is added to $\left|u_{\text {red }}\right\rangle$ by transporting the tail of $\left|u_{\text {green }}\right\rangle$ so that it falls upon the head of $\left|u_{\text {red }}\right\rangle$. The sum $|\psi\rangle$ is a vector drawn from the origin to the transported head of $\left|u_{\text {green }}\right\rangle$. 
These coefficients express the probabilities of the alternative outcome states in the following way: $\alpha$ and $\beta$ are "projections" of the vector $|\psi\rangle$ upon the eigenvector $\left|u_{\text {green }}\right\rangle$ and the eigenvector $\left|u_{\text {red }}\right\rangle$ respectively (see figure 3.4). So, $\alpha$ is the length of the projection of $|\psi\rangle$ upon $\left|u_{\text {green }}\right\rangle$, and this length represents the probability of $\left|u_{\text {green }}\right\rangle$ becoming actual. The projection of $|\psi\rangle$ upon $\left|u_{\text {green }}\right\rangle$ is written as:

$$
\alpha=\left\|\mathrm{P} u_{\text {green }} \psi\right\|
$$

Similarly, $\beta$ is the length of the projection of $|\psi\rangle$ upon $\left|u_{\text {red }}\right\rangle$, written as:

$$
\beta=\left\|\mathrm{P} u_{\text {red }} \psi\right\|
$$

As one can see by looking at figure $3.4,|\alpha|^{2}+|\beta|^{2}=1$ is simply the necessary satisfaction of the Pythagorean theorem by the projectors; and as these coefficients are intended to express probabilities,

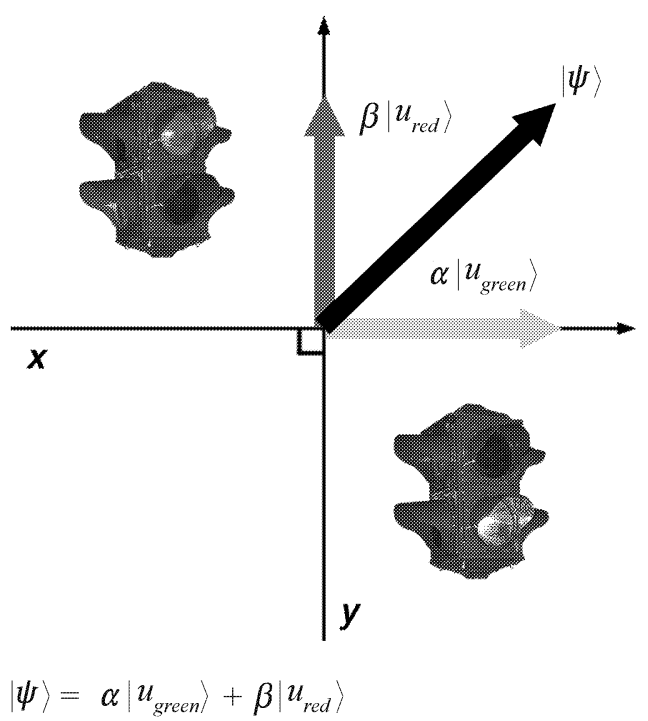

FIgURE 3.3. The pure state $|\psi\rangle$ as an objectively real, but objectively indefinite superposition of states $\left|u_{\text {green }}\right\rangle$ and $\left|u_{\text {red }}\right\rangle$, expressed as the sum of $\left|u_{\text {green }}\right\rangle$ and $\left|u_{\text {red }}\right\rangle$, with the complex coefficients satisfying $|\alpha|^{2}+|\beta|^{2}=1$. This sum to unity of the probable outcome states represented by $\left|u_{\text {green }}\right\rangle$ and $\left|u_{\text {red }}\right\rangle$ thus accords with standard probability theory; $\alpha\left|u_{\text {green }}\right\rangle$ and $\beta\left|u_{\text {red }}\right\rangle$ are probability-valuated alternative outcome states. 


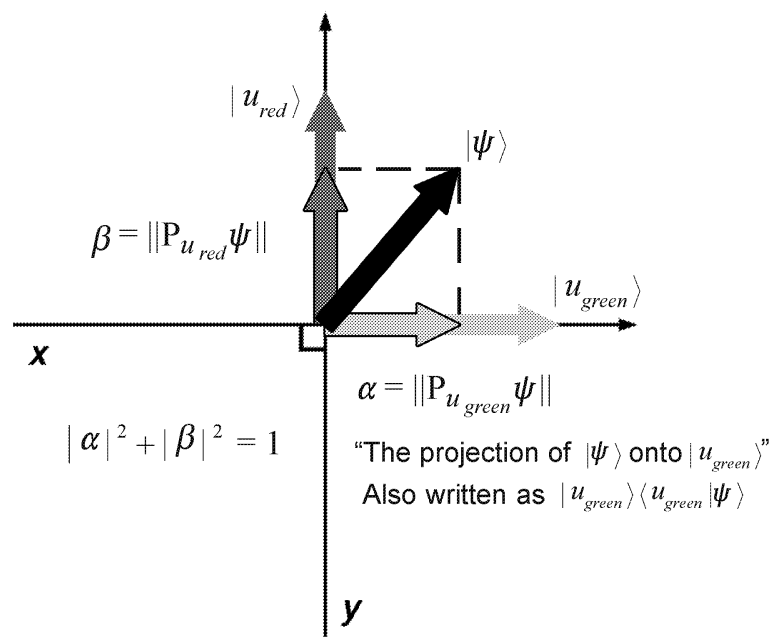

Figure 3.4. The complex coefficient $\alpha$ is the length of the projection of $|\psi\rangle$ upon $\left|u_{\text {green }}\right\rangle$, and the complex coefficient $\beta$ is the length of the projection of $|\psi\rangle$ upon $\left|u_{\text {red }}\right\rangle$.

$|\alpha|^{2}+|\beta|^{2}=1$ also satisfies standard probability theory. It is significant, however, that in quantum mechanics, neither $\alpha\left|u_{\text {green }}\right\rangle$ nor $\beta\left|u_{\text {red }}\right\rangle$ are vectors of unit length, such that neither represents a system state by itself; only when added together, correlated, do they sum to unity, thus representing a valid state. This is the infamous "Superposition Principle."

This correlated, pure state $|\psi\rangle=\alpha\left|u_{\text {grenn }}\right\rangle+\beta\left|u_{\text {red }}\right\rangle$ evolves to become a mixed state where the correlated superposition of eigenstates $\alpha\left|u_{\text {green }}\right\rangle+\beta\left|u_{\text {red }}\right\rangle$ is now expressible as a "density matrix" $\rho$ of separate, uncorrelated mutually exclusive and exhaustive outcomes, each valuated as a probability.

As discussed earlier, quantum mechanics does not describe the evolution of the mixed state, to a unique state $\left|u_{\text {green }}\right\rangle$ or $\left|u_{\text {red }}\right\rangle$; quantum mechanics describes only the evolution of the much broader "pure state" superposition $|\psi\rangle=\alpha\left|u_{\text {green }}\right\rangle+\beta\left|u_{\text {red }}\right\rangle$ to the mixed state. Put another way, quantum mechanics does not include a mechanism for the actualization of potentia; it merely describes the valuation of potentia (via the complex coefficients $\alpha$ and $\beta$ ) -the valuation of the alternative potential eigenstates belonging to the mixed state, such that these alternative potential states become probabilities, and not just potentia. 


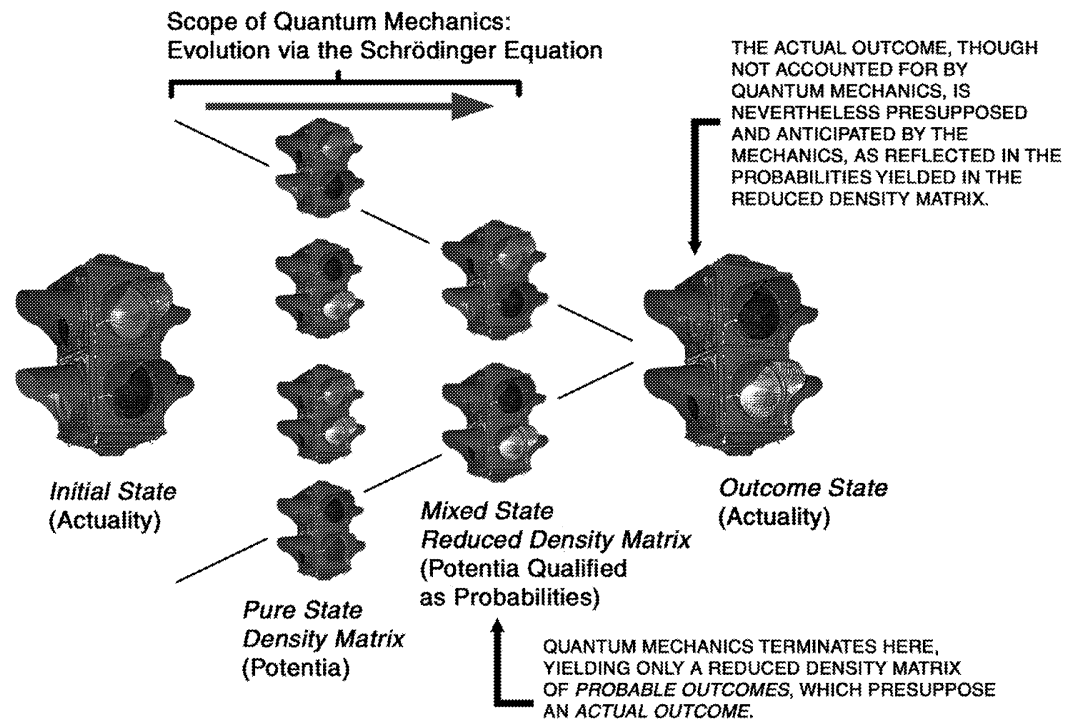

Figure 3.5. The quantum mechanical evolution of an idealized trafficsignal system.

The first, most obvious ontological issue raised by this mathematical, linear vector description of quantum mechanics concerns the expression of the initial state vector $|\psi\rangle$ as a sum of the potential outcome state eigenvectors. Even in our idealized traffic-light example, $|\psi\rangle$ can clearly be expressed as the sum of a great many orthogonal vectors other than $\left|u_{\text {green }}\right\rangle$ and $\left|u_{\text {red }}\right\rangle$. Why does the initial state vector $|\psi\rangle$ always evolve to become $\left|u_{\text {green }}\right\rangle$ or $\left|u_{\text {red }}\right\rangle$, rather than some other set of orthogonal vectors, representing other mutually exclusive and exhaustive outcome states, such as, for example, $\left|u_{\text {purple }}\right\rangle$ or $\left|u_{\text {hazel }}\right\rangle$ ? Why, in other words, is Schrödinger's cat necessarily a live or dead cat when the box is opened, and not a mouse or a dog?

From a strictly instrumentalist interpretation of quantum mechanics, this somewhat arbitrary selection of alternative mutually exclusive and exhaustive outcome states and their associated mutually orthogonal state vectors (referred to as the "preferred basis" or "pointer basis") poses no great problem, inasmuch as the alternative potential outcome states are always known prior to measurement. In other words, we simply know our traffic signal will either be red or 
green upon measurement, and not purple, hazel, aquamarine, and so on. As we will see later in this chapter, however, the ontological significance of the preferred basis really derives from the fact that a quantum mechanical system state always evolves relative to a particular quantum fact (the "indexical eventuality") associated with the apparatus performing the measurement. The preferred basis, in other words, is a feature of this apparatus and its contribution to the evolution of the system state. The ineluctability of this relativity implies, as von Neumann suggested, that the measuring apparatus together with the system measured should be interpreted as a composite quantum system. As the measured system evolves throughout a measurement interaction, then, the measuring apparatus evolves along with it; facts constitutive of one must correlate with facts constitutive of the other.

An equally significant question, though, is this: Why can't $|\psi\rangle$ evolve to become a density matrix of nonorthogonal vectors- that is, vectors representing nonsensical states that are not mutually exclusive and that "interfere" with each other-states whose vectors belong neither to $\mathscr{E}$ nor to $\mathscr{E}^{\perp}$ for a given fact? Such states would subsume facts that are incapable of integration, such as location in two places at once, or a cat being both alive and dead at the same time. Why doesn't one ever see such superpositions? An ontological interpretation of quantum mechanics must, it would seem, allow for their existence, since it is the evolution of these superpositions of ontologically significant potentia that quantum mechanics describes. If these potentia don't "really exist," what, exactly, is evolving mechanically? An instrumentalist would likewise answer that the indefiniteness of the initial state $|\psi\rangle$ is merely epistemological; for even if $|\psi\rangle$ could in theory evolve to become a sum of nonorthogonal vectors representing mutually interfering nonsensical states, the question as to whether or not such states can ever exist is seldom entertained in the lab, since they have never been encountered.

If, however, one seeks to explore the ontological significance of the conceptual innovations and implications of quantum mechanics - and there is clearly no reason not to do so, given that quantum mechanics has proven to be the most accurate and thus far the most fundamental description of nature yet conceived-one must be able to adequately answer these questions, and others like them. To that 
end, let us revisit the notion that quantum mechanics evinces two fundamental species of reality-actualities and potentia. Recall that for Heisenberg, the concept of potentia serves as "a first definition concerning the ontology of quantum theory. . . One sees at once that this use of the word 'state,' especially the term 'coexistent state,' is so different from the usual materialistic ontology that one may doubt whether one is using a convenient terminology. . . One may even simply replace the term 'state' by the term 'potentiality' - then the concept of 'coexistent potentialities' is quite plausible, since one potentiality may involve or overlap other potentialities." 1

In our red-green traffic-signal example, the pure state represented by $|\psi\rangle$ can be expressed as the sum of a practically infinite number of vectors. Some of these vectors are mutually orthogonal, representing mutually exclusive potential states, and some are nonorthogonal, representing interfering or nonsensical potential states. All of these potential states are, in a sense, englobed by $|\psi\rangle$, such that $|\psi\rangle$ can be thought of as representing a sort of infusion of pure potentiality into the antecedently "actual" state of the system as it evolves. For it must not be overlooked that our traffic-signal system exists in some "actual" state prior to measurement-namely, $\left|u_{\text {green }}\right\rangle$ or $\left|u_{\text {red }}\right\rangle$-just as it will again, once its evolution, through $|\psi\rangle$, is satisfied. If, for example, our traffic signal were initially green (i.e., "prepared" in a green state prior to measurement), then $|\psi\rangle$ represents the actual state $\left|u_{\text {green }}\right\rangle$ now augmented with a pure potentiality, represented in the formalism by the potential projection of $|\psi\rangle$ upon a practically infinite number of vectors in a practically infinite number of dimensions, representing a practically infinite number of system statessome sensible, others nonsensical and seemingly impossible.

With respect to such states, Murray Gell-Mann once wrote of being assigned, as an undergraduate, the problem of using quantum mechanics to calculate the probability that a heavy macroscopic object would, during a given time interval, jump a foot into the air-a ridiculous state nevertheless subsumed by $|\psi\rangle$. "The answer," GellMann wrote, "was around one divided by the number written as one followed by sixty-two zeros"2_an exceedingly improbable state, for all practical purposes impossible, yet far from ontologically insignificant; for the potentiality of similar, exceedingly improbable states is precisely why useful effects such as quantum tunneling are possible, 
and why devices that make use of these effects, such as the scanning tunneling microscope (STM), are able to function.

Returning to our idealized traffic-light system, then, it must be stressed that both $\left|u_{\text {green }}\right\rangle$ and $|\psi\rangle$ refer to the same "real" system, with the latter, potentiality-infused state $|\psi\rangle$ evolving from the former "actual" state $\left|u_{\text {green }}\right\rangle$. The systems represented by $\left|u_{\text {green }}\right\rangle$ and $|\psi\rangle$ and their evolution, one to the other, are, in other words, ontologically significant by this interpretation. According to quantum mechanics, it is the nature of such system states to evolve, and the concept of evolution, like the concept of probability, always presupposes an actual beginning and an actual end-a system in its initial state and a system in its final state-the probability of $S_{\text {initial }}$ becoming $S_{\text {final }}$. But evolution and probability also presuppose actuality and potentiality; both states, initial and final, are actualities-collections of facts, the former giving rise to the latter via an integration of potentia.

The primary phase of the quantum mechanical evolution of a state vector, then, is the evolution of some actual state-either $\left|u_{\text {green }}\right\rangle$ or $\left|u_{\text {red }}\right\rangle$ in our traffic light example - to the pure state $|\psi\rangle$, which like $\left|u_{\text {green }}\right\rangle$ or $\left|u_{\text {red }}\right\rangle$ is a "real" state of the system; but unlike $\left|u_{\text {green }}\right\rangle$ or $\left|u_{\text {red }}\right\rangle,|\psi\rangle$ can be projected onto a practically infinite number of vectors, both mutually orthogonal and nonorthogonal, thus englobing every potential state - both sensible and nonsensical-into which our traffic light might evolve.

At this point in the discussion, it will be useful to alter our trafficsignal example slightly so that it better resembles a typical quantum mechanical measurement interaction. To the traffic-signal system $S$, we shall add a detector system $D$, which is the instrument by which we are able to measure the color of the traffic signal. $S$ and $D$ together constitute a composite system whose combined Hilbert spaces form a tensor product space such that $S$ and $D$ together will evolve in a correlated manner, no differently than $S$ alone as described thus far; in fact, $S$ and $D$ can just as well be considered individual subsystems or even individual facts of a larger system $U$ (the universe). The dividing line separating the measured system from the measuring apparatus and environment is, as discussed earlier, purely arbitrary and a matter of convenience.

Let us suppose that our detector $D$ is a simple pointer instrument, 
whose needle will point up in a state $\left|d_{\uparrow}\right\rangle$ if the traffic signal is red, and down in a state $\left|d_{\downarrow}\right\rangle$ if the traffic signal is green. Thus, the Hilbert space $\mathscr{H}_{D}$ representing our detector is, like $\mathscr{H}_{S}$, two-dimensional, and both spaces together form a single, tensor product space in which the evolution of our initial composite system $\left|\phi^{i}\right\rangle$ will be described.

Recall that without the detector, the pure state of $S$ was described as:

$$
|\psi\rangle=\alpha\left|u_{\text {green }}\right\rangle+\beta\left|u_{\text {red }}\right\rangle
$$

Now, we have a composite, initial $S$ and $D$ system $\left|\phi^{i}\right\rangle$ whose pure state similarly evolves as:

$$
\begin{aligned}
\left|\phi^{i}\right\rangle & =\left(\alpha\left|u_{\text {green }}\right\rangle+\beta\left|u_{\text {red }}\right\rangle\right)\left|d_{\downarrow}\right\rangle \\
& \Rightarrow \alpha\left|u_{\text {green }}\right\rangle\left|d_{\downarrow}\right\rangle+\beta\left|u_{\text {red }}\right\rangle\left|d_{\uparrow}\right\rangle=\left|\phi^{c}\right\rangle
\end{aligned}
$$

This evolution from the initial composite state $\left|\phi^{i}\right\rangle$ to the pure, correlated composite state $\left|\phi^{c}\right\rangle$ is described by the Schrödinger equation and constitutes the essence of a quantum mechanics; and from a practical standpoint, the correlated composite state $\left|\phi^{c}\right\rangle$ is entirely

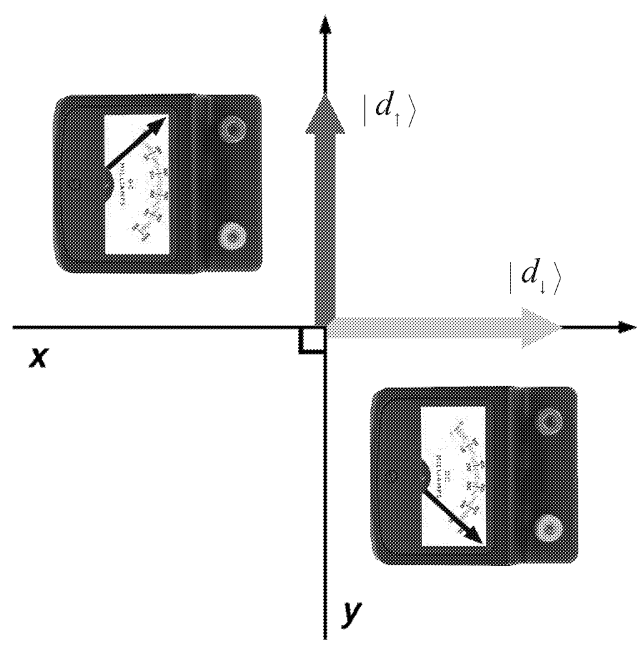

Figure 3.6. The two potential states $D$ of a traffic-signal detector, represented by orthogonal vectors of unit length in a Hilbert space of two dimensions. The vector $\left|d_{\downarrow}\right\rangle$ along the $x$-axis represents " $D=$ green" and the vector $\left|d_{\uparrow}\right\rangle$ along the $y$-axis represents " $D=$ red." 

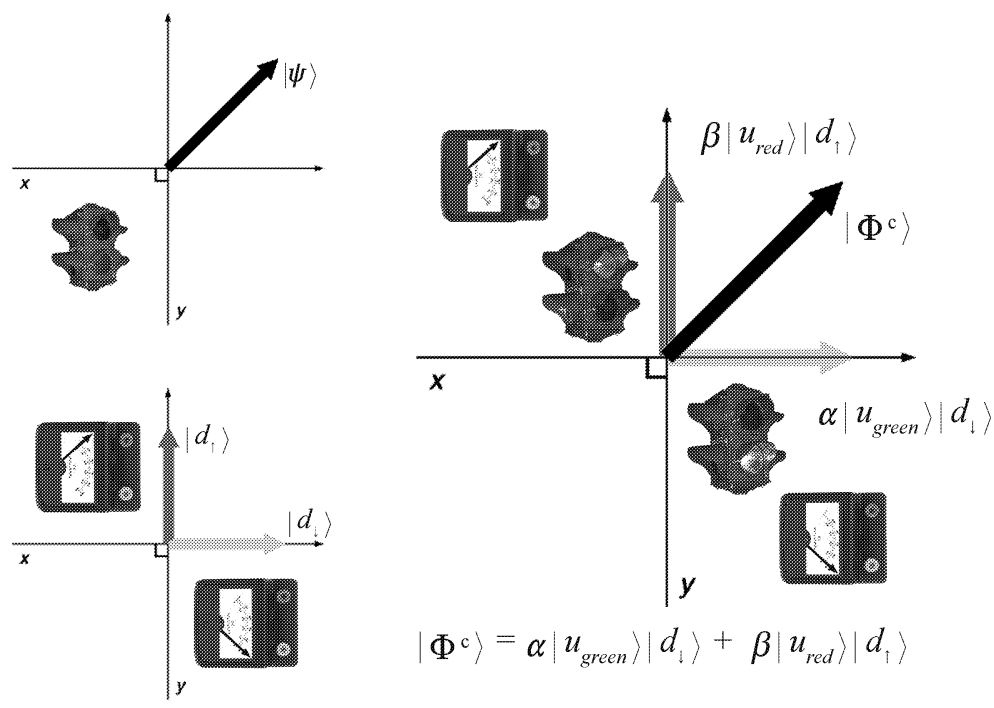

FigurE 3.7. The composite, correlated system SD represented by the vector $\left|\Phi^{c}\right\rangle$.

satisfactory, since the two mutually exclusive and exhaustive, probability-valuated alternative states it subsumes, $\alpha\left|u_{\text {green }}\right\rangle\left|d_{\downarrow}\right\rangle$ and $\beta\left|u_{\text {red }}\right\rangle\left|d_{\uparrow}\right\rangle$, are both sensible: Whenever the signal is green, our detector points down, and whenever the signal is red, our detector points up. A probability is assigned to each of these alternative states, reflected in the density matrix:

$$
\rho^{\mathrm{r}}=|\alpha|^{2}\left|u_{\text {green }}\right\rangle\left\langle u_{\text {green }}|| d_{\downarrow}\right\rangle\left\langle\left. d_{\downarrow}|+| \beta\right|^{2} \mid u_{\text {red }}\right\rangle\left\langle u_{\text {red }}|| d_{\uparrow}\right\rangle\left\langle d_{\uparrow}\right|
$$

and repeated measurements will always agree with those probability valuations.

But from an ontological standpoint, we have the same difficulties discussed earlier:

(i) Why does the density matrix of the mixed state always subsume sensible, mutually exclusive and exhaustive alternative states? Why can't the mixed state ever subsume nonsensical states such as $\alpha\left|u_{\text {green }}\right\rangle\left|d_{\uparrow}\right\rangle$ or superpositions of states?

(ii) Superpositions aside, why does the pure state always evolve to the preferred basis of the detector in the mixed state, rather than some other potential state? 
With respect to the latter question, it was discussed earlier that quantum mechanics does not merely describe the mutual interrelations among all facts comprised by a given system or composite system; quantum mechanics describes, rather, the evolution of the state of a system relative to a particular fact (or subsystem) belonging to the composite system-or, more accurately, relative to the interrelations between this particular fact or subsystem and all other facts belonging to the measured system. In quantum mechanics, this "particular fact or subsystem"-referred to as the "indexical eventuality"always belongs to the measuring apparatus. Thus, the mixed state always reflects the preferred basis of the measuring apparatus, since the state of the system evolves relative to the indexical eventuality belonging to this apparatus. The grouping of potential state vectors into subspaces $\mathscr{E}$ and $\mathscr{E}^{\perp}$ exemplifies this relativity, where all potential states sharing the indexical eventuality $\left|d_{\uparrow}\right\rangle$, for example, belong to $\mathscr{E}$ and all others belong to $\mathscr{E}^{\perp}$. It is this evolution of the pure state relative to a particular fact (or subsystem) that conditions the elimination of superpositions of interfering, "coherent" states via a process of negative selection. The only potential states that remain-those constituting the mixed state-are, as a result, noninterfering, "decoherent" potential states, which have been valuated as probabilities.

Before exploring the mechanics of this elimination via negative selection, effected by the state's evolution relative to a particular fact, it should be pointed out that this mechanism is often described more simply as follows: "Superpositions are eliminated by the measuring apparatus as it interacts with the system measured." Heisenberg, for example, writes that in the quantum formalism, the superposition or interference of potentia, "which is the most characteristic phenomenon of quantum theory, is destroyed by the partly indefinable and irreversible interactions of the system with the measuring apparatus and the rest of the world."3 The difficulty of this wording, with its arbitrary separation of the universe into system, apparatus, and "the rest of the world" (environment), is the intrusion of sheer subjectivity it implies; for as we saw earlier, the final "measuring apparatus" in the von Neumann chain of apparatuses is, unavoidably, the mind of the human observer. In defense against this implication, Heisenberg states: 
Certainly quantum theory does not contain genuine subjective features, it does not introduce the mind of the physicist as part of the atomic event. But it starts from the division of the world into the "object" and the rest of the world. ... This division is arbitrary and historically a direct consequence of our scientific method; the use of classical concepts is finally a consequence of the general human way of thinking. ${ }^{4}$

Since, however, we are here exploring an ontological rapprochement of the classical, materialistic worldview and a worldview capable of accommodating quantum mechanics, it is important to acknowledge explicitly the following two points: (i) the universe, since it is the only truly closed system, is the only system to which quantum mechanics can properly apply if quantum mechanics is to be thought of as being ontologically significant; and (ii) the grouping of various facts within this system into composite subsystems of "system," "apparatus," and "environment" subsystems is purely arbitrary. Given these two points, one should likewise acknowledge that the evolution of the state of a measured system is, in fact, the evolution of the state of the universe itself; therefore, its evolution relative to a given "measuring apparatus" is merely its evolution relative to a particular fact (or collection of facts) belonging to itself. In the same sense, the distinction between a single fact and a subsystem of facts within the universe is arbitrary as well, and this applies to the states $S$ (system), $D$ (detector), and $E$ (environment). The correlation among these subsystems is easily comprehended by virtue of the fact that as the closed system of the universe evolves, so must all the facts subsumed by it, however they might be grouped and whatever they might be named. These last two points are clearly expressed by Heisenberg in the following:

The measuring device ... contains all the uncertainties concerning the microscopic structure of the device which we know from thermodynamics, and since the device is connected with the rest of the world, it contains in fact the uncertainties of the microscopic structure of the whole world.

These ontological implications aside, however, the quantum mechanical description of a measurement interaction, such as the one in our example, is typically left at:

$$
\begin{aligned}
\left|\Phi^{\mathrm{i}}\right\rangle & =\left(\alpha\left|u_{\text {green }}\right\rangle+\beta\left|u_{\text {red }}\right\rangle\right) \mid d_{\downarrow} \\
& \Rightarrow \alpha\left|u_{\text {green }}\right\rangle\left|d_{\downarrow}\right\rangle+\beta\left|u_{\text {red }}\right\rangle\left|d_{\uparrow}\right\rangle=\left|\Phi^{\mathrm{c}}\right\rangle
\end{aligned}
$$


since this expression provides sensible outcome states, each of which is accurately valuated as a probability, such that successful predictions can be made. The question as to the mechanism of negative selection by which the superpositions of interfering states in $|\psi\rangle$ are eliminated from the above expression is a question which begs the ontological significance of quantum mechanics, and thus has received relatively little attention in physics.

\section{DeCoherence}

The persisting lack of a truly coherent ontology capable of accommodating quantum mechanics, however, has throughout recent years inspired a number of physicists to examine more closely this mechanism of negative selection by which quantum superpositions are eliminated. It was stated earlier that the key to this mechanism, for certain physicists, was to be found in the very desiderata required by a coherent ontological interpretation of quantum mechanics, with the most significant of these being that the interpretation be universally applicable. The closed system in which every quantum mechanical measurement takes place must, at a fundamental level, be understood to be the universe itself; and from that stipulation, it must be acknowledged that the environment englobing a measured system (i.e., a measured subsystem of the universe) - though it may be and typically must be ignored for the practical purposes of calculation-must therefore play some role in the evolution of the state of the measured system. Many theorists, including Wojciech Zurek, Robert Griffiths, Roland Omnès, James Hartle, and Murray GellMann, among several others, have demonstrated that the interrelations between "measured" facts and facts belonging to the "environment" englobing the measurement interaction play a crucial mechanical role in the elimination of superpositions via negative selection. Though the proposals of each of these thinkers differ somewhat, they all emphasize the importance of this negative selection process, and mutually refer to the result of this process as the "decoherence effect."

In other words, the correlated superposition

$$
\left|\Phi^{c}\right\rangle=\alpha\left|u_{\text {green }}\right\rangle\left|d_{\downarrow}\right\rangle+\beta\left|u_{\text {red }}\right\rangle\left|d_{\uparrow}\right\rangle
$$


evolves via a process of negative selection to become a mixed state of decoherent, probability-valuated, mutually exclusive and exhaustive alternative states in the reduced density matrix:

$$
\rho^{r}=|\alpha|^{2}\left|u_{\text {green }}\right\rangle\left\langle u_{\text {green }}|| d_{\downarrow}\right\rangle\left\langle\left. d_{\downarrow}|+| \beta\right|^{2} \mid u_{\text {red }}\right\rangle\left\langle u_{\text {red }}|| d_{\uparrow}\right\rangle\left\langle d_{\uparrow}\right|
$$

Furthermore, these theorists agree that the negative selection mechanism by which decoherence is effected involves the environment of the system measured-though they disagree slightly as to how, exactly, the environment functions in this regard. The extent of this disagreement lies beyond the scope of this essay, however, so for the current discussion it will suffice to focus on just one of these decoherence interpretations-the Environmental Superselection interpretation of Wojciech Żurek. ${ }^{6}$

"Decoherence results from a negative selection process that dynamically eliminates nonclassical states," writes Zurek, who maintains that decoherence is a consequence of the universe's role as the only truly closed system-which, put another way, guarantees the ineluctable "openness" of every subsystem within it. "This consequence of openness is critical in the interpretation of quantum theory," Zurek continues, "but seems to have gone unnoticed for a long time." The key to understanding the negative selection mechanism of decoherence in Zurek's interpretation is the supplemental process, suggested by von Neumann and introduced in chapter $2^{8}$ - his "Process 1"- whereby the reduced density matrix of the mixed state evolves from a larger, correlated, pure state density matrix. This larger, pure state density matrix, unlike the reduced matrix, contains nonsensical, coherent states (noted in boldface) which must be eliminated:

$$
\begin{aligned}
\rho^{c}= & |\alpha|^{2}\left|u_{\text {green }}\right\rangle\left\langle u_{\text {green }}|| d_{\downarrow}\right\rangle\left\langle d_{\downarrow}\left|+\boldsymbol{\alpha} \boldsymbol{\beta}^{*}\right| \boldsymbol{u}_{\text {green }}\right\rangle\left\langle\boldsymbol{u}_{\text {red }} \mid d_{\downarrow}\right\rangle\left\langle d_{\uparrow}\right| \\
& +\boldsymbol{\alpha}^{*} \boldsymbol{\beta}\left|\mathbf{u}_{\text {red }}\right\rangle\left\langle\mathbf{u}_{\text {green }} \mid \mathbf{d}_{\uparrow}\right\rangle\left\langle\left.\mathbf{d}_{\downarrow}|+| \beta\right|^{2} \mid u_{\text {red }}\right\rangle\left\langle u_{\text {red }}|| d_{\uparrow}\right\rangle\left\langle d_{\uparrow}\right|
\end{aligned}
$$

This evolution of the state vector from the correlated pure state $\left|\phi^{c}\right\rangle$ and its pure state density matrix $\rho^{c}$ to the mixed state and its reduced density matrix $\rho^{r}$ is useful, in that one is able to account for the elimination from $\rho^{c}$ of the nonsensical, correlated states that are incapable of integration in the reduced density matrix. The "offdiagonal" terms in boldface represent these nonsensical states, 
whose vectors are mutually nonorthogonal. Typically, one merely cancels out these off-diagonal terms, yielding the reduced density matrix; however, the ontological interpretations that make use of the decoherence effect suggest an ontologically significant process by which the interaction of the measured system with its measuring apparatus and its environment produces the necessary cancellations.

In Zurek's Environmental Superselection interpretation, ${ }^{9}$ the interaction of the environment $\left|E_{0}\right\rangle$ with $\left|\phi^{c}\right\rangle$ is described as:

$$
\begin{aligned}
\left|\Phi^{c}\right\rangle\left|E_{0}\right\rangle & =\left(\alpha\left|u_{\text {green }}\right\rangle\left|d_{\downarrow}\right\rangle+\beta\left|u_{\text {red }}\right\rangle\left|d_{\uparrow}\right\rangle\right)\left|E_{0}\right\rangle \\
& \Rightarrow \alpha\left|u_{\text {green }}\right\rangle\left|d_{\downarrow}\right\rangle\left|E_{\downarrow}\right\rangle+\beta\left|u_{\text {red }}\right\rangle\left|d_{\uparrow}\right\rangle\left|E_{\uparrow}\right\rangle=|\Psi\rangle
\end{aligned}
$$

This correlation of the environment, the system measured, and the detector, and the cancellations of interfering nonorthogonal potential states it produces can most easily be visualized by recalling the simple concept of subspaces: The subspace $\mathscr{E}_{\downarrow}$ contains all vectors representing all potential states of the universe where our detector's

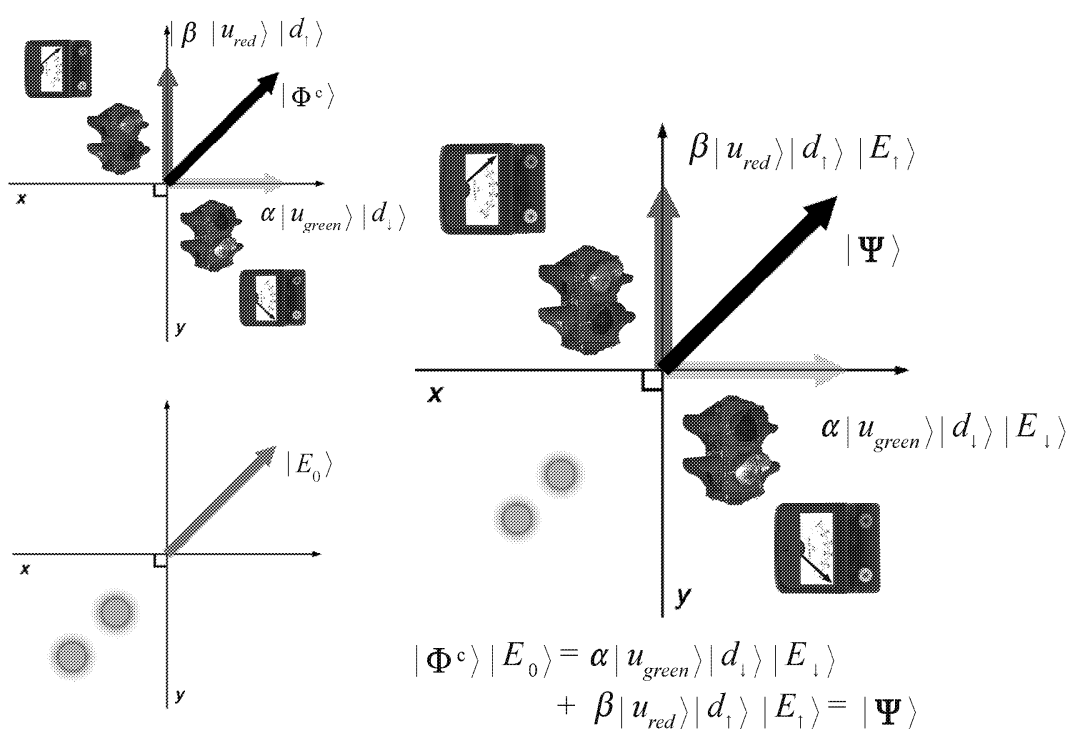

Figure 3.8. The composite system SD is correlated with the Environment (E), forming a new correlated, composite system SDE, represented by the vector $|\Psi\rangle$. 
pointer is down: $\left|d_{\downarrow}\right\rangle$. Recall that a state is a maximal specification of all facts comprised by a system, so that every potential state vector belonging to $\mathscr{E} \downarrow$ represents a different, potential "snapshot" of the universe and all its facts. But in $\mathscr{E} \downarrow$ all of these snapshots have at least one fact in common: Our detector's pointer is down. Likewise, we have a subspace consisting of all vectors orthogonal to $\mathscr{E} \downarrow$ and in this subspace $\mathscr{E}_{\downarrow}^{\perp}$ the fact common to all vectors belonging to it is that our detector's pointer is not down.

Because the environment subsumes a nearly infinite number of potential facts, its correlation with our system-detector $\left|\phi^{c}\right\rangle$ produces a practically infinite number of degrees of freedom-different potential projections of $|\Psi\rangle$ upon $\mathscr{E}_{\downarrow}$ and $\mathscr{E}_{\downarrow}{ }^{\perp}$, and $\mathscr{E}_{\uparrow}$ and $\mathscr{E}_{\uparrow}{ }^{\perp}$, producing a practically infinite number of potential state vectors, some of which belong to $\mathscr{E}_{\downarrow}$ and some of which belong to $\mathscr{E}_{\downarrow}^{\perp}$.

This unimaginably large number of potential state vectors in $\mathscr{E} \downarrow$ and $\mathscr{E}_{\downarrow}^{\perp}$ is useful because it produces a great deal of cancellation of interfering, incompatible states within each of these subspaces.

In an ontological interpretation of quantum mechanics for which logical consistency is a desideratum, the justification for this conception of 'interfering, incompatible states' and the elimination of such states is simply a matter of satisfying the logical principles of noncontradiction and the excluded middle. While it is true that many physicists tend to dismiss such ontological justifications as unscientific, metaphysical hand-waving, it must be remembered that the correlation of causal relation and logical implication, central to modern science, is a presupposed correlation; it is, in other words, an ontological 'first principle' of science. Since modern science is impossible without this presupposition of logical causality, the appeal to logical causality as an ontological desideratum of quantum mechanics would seem entirely justified as a necessary and sufficient principle to account for the elimination of superpositions of interfering states.

Consider, for example, the following two potential states of $|\Psi\rangle$ belonging to $\mathscr{E}_{\downarrow}$ :

(i) A state where our detector points down, our traffic signal is green, and a particular photon from the sun strikes the green lens.

(ii) A state where our detector points down, our traffic signal is red, and this particular photon strikes the red lens. 
These two potential states are interfering states, incapable of integration, as are the vast majority of states in $\mathscr{E}_{\downarrow}$ and $\mathscr{E}_{\downarrow}{ }^{\perp}$, and serve to cancel each other out. In our experiment, we are concerned only with the correlation of the detector and the traffic signal, such that we ignore all other potential facts in the universe, including the status of photons from the sun as they strike the traffic signal. But because these and the multiplicity of other facts belonging to the environment are correlated with our system and detector, the cancellations they produce also eliminate the interferences we are concerned with, such as our detector pointing down and our traffic signal being red instead of green.

Put another way, the Hilbert spaces describing our traffic signal and detector by themselves span only two dimensions, which are not sufficient to produce the cancellations needed to eliminate unwanted superpositions of interfering states. By correlating our system and detector with the environment and its Hilbert space of practically infinite dimensions, we are able to produce enough potential states of $|\Psi\rangle$ such that most of these are mutually interfering and self-cancelling, thereby eliminating superpositions of interfering states.

This cancellation is represented mathematically as a "trace-over" of the uncontrolled and unmeasured degrees of freedom contributed by the environment, and the density matrix that results from this cancellation is written as:

$$
\rho_{\mathrm{SD}} \equiv \operatorname{Tr}_{E}|\Psi\rangle\langle\Psi|=\Sigma_{i}\left\langle E_{i} \mid \Psi\right\rangle\left\langle\Psi \mid E_{i}\right\rangle=\rho^{r}
$$

with $\rho^{r}$ here being identical to the reduced density matrix we had earlier, before introducing the environmental correlations: ${ }^{10}$

$$
\rho^{r}=|\alpha|^{2}\left|u_{\text {green }}\right\rangle\left\langle u_{\text {green }}|| d_{\downarrow}\right\rangle\left\langle\left. d_{\downarrow}|+| \beta\right|^{2} \mid u_{\text {red }}\right\rangle\left\langle u_{\text {red }}|| d_{\uparrow}\right\rangle\left\langle d_{\uparrow}\right|
$$

Thus, the interrelation of environmental facts with the facts comprised by our system and detector continuously reduces any coherent, interfering superpositions of states relative to the preferred basis $\left|d_{\downarrow}\right\rangle$ and $\left\langle d_{\uparrow}\right|$, such that the only alternative states in the reduced density matrix are mutually exclusive and exhaustive, logical states, each valuated according to a probability. "An effective superselection rule has emerged," Zurek writes. "Decoherence prevents superpositions of the preferred basis states from persisting. Moreover, we have obtained all this—or so it appears—without having to appeal 
to anything beyond the ordinary, unitary Schrödinger evolution."11 (See Figure 3.9 in Appendix.)

The ontological significance of this interpretation of quantum mechanics, with its mechanism of negative selection of interfering potentia via the decoherence effect, becomes acute when one explores the cosmological implications-especially since this interpretation of quantum mechanics explicitly recognizes that all facts subsumed by the closed system of the universe are mutually interrelated in any given measurement interaction. Many theorists have pursued these implications, and in so doing have suggested a more intuitive and more generalized way of describing the evolution of states of the universe: Instead of focusing on states (akin to "snapshots" of the actualities of the universe), these theorists focus on "histories," or historical routes of states-that is, histories of facts whose perpetual augmentation by novel facts is presupposed.

The elimination of interfering potentia and the probability-valuation of those that remain, via the environmental correlations and trace-over described above, is thus seen as the elimination of interfering potential histories. Separate systems with separate histories, by virtue of their correlation with a shared environment (i.e., the universe), necessarily entail many shared potential facts. And since these shared potential facts must be mutually consistent, so must those histories which include them-lest nature become overrun with violations of the logical principle of noncontradiction (physical bodies being two places at once, etc.) - the fundamental principle which bridges the logical realm of the necessary and the causal realm of the contingent. Interfering potential histories, those that are not mutually exclusive and exhaustive, are thus eliminated by integration via environmental correlation. Thus, the mutual historical consistency of multiple systems, even those which are spatially well separated, derives in large part from their being englobed by a larger, shared "environmental history."

This concept of decoherent, consistent histories associated with environmental correlations is central to the quantum mechanical interpretations of Robert Griffiths, ${ }^{12}$ Roland Omnès, ${ }^{13}$ and Murray Gell-Mann and James Hartle. ${ }^{14}$ In all of these interpretations, the term "history" implies an ongoing process not necessarily connoted in the term "state." For in quantum mechanics, as we have seen, it is the nature of a state to evolve continuously in one sense, yet discontinuously in another, just as a history of actual events evolves; 
thus, the evolution of both "state" and "history" is characterized as fluid potentiality punctuated by an ever-expanding lineage of discrete actualities/events/facts, each novel actuality being subsequent to and partially consequent of all actualities antecedent to it. And just as the concepts of probability and the evolution of a state both imply an actual past and a presupposed, subsequent, and consequent actual future, so does the concept of a "history."

Thus, we may characterize the alternative states of the reduced density matrix of any given measurement interaction as "alternative potential histories" of the universe. This set of histories is understood to be interrelated with all antecedent facts belonging to the universe, and it is this interrelation which allows for a subsequent elimination of the vast majority of potentia produced by this interrelation, such that interfering superpositions of potential historieshistories incompatible for integration in the reduced density matrix - are eliminated. Those histories that remain are referred to as "consistent histories"- the definition of which, as applied to quantum mechanics, was first proposed by Robert Griffiths, ${ }^{15}$ who demonstrated that not only integrations of alternative potential states but also sequences or histories of alternative potential states can eliminate interfering states. Griffiths thus demonstrated the equivalence between alternative states and alternative histories of states valuated as mutually exclusive and exhaustive probabilities in the reduced density matrix. Gell-Mann and Hartle have shown that Griffiths's "consistency conditions" are similar to those required for the decoherence effect, with the conditions for the latter being slightly stronger, in fact (hence, their use of the term "strong decoherence," discussed in their paper of the same name ${ }^{16}$ ).

\section{Three Benefits of This Ontological INTERPRETATION OF THE QUANTUM FORMALISM}

Though there are a great many benefits to the ontological interpretation of quantum mechanics presented thus far, three stand out at this point in the discussion. First, this interpretation is a truly universal interpretation of quantum mechanics. Unlike models based upon Bohr's notion of complementarity, with its arbitrary divisions of the world into "subject" and "object," each with its own body of physical laws incompatible with those of the other, the decoherence-based 
interpretations require no such division; rather, these interpretations make use of the principle that facts belonging to "subject," "object," and "environment" are necessarily ontologically interrelated. These interrelations give rise, via the Schrödinger equation, to manifold logical integrations of potential states which, via nothing other than the process of integration itself, eliminate superpositions of interfering states incompatible for integration. The decoherence-based interpretations thereby purge quantum mechanics of its infamous predication of objective reality upon subjective-and by some interpretations, necessarily human-observations. It is because of this first benefit that decoherence-based interpretations of quantum mechanics are often described as "quantum mechanics without observers." Of course, this characterization is not entirely accurate insofar as "observation" by this interpretation is described as the interrelation among all facts subsumed by a system-that is, observer-facts interrelated with measured system-facts and environmental facts. The advantage of the decoherence-based interpretations is that they explicitly acknowledge and make use of the concept that the universe itself is the only truly closed system, and therefore all facts englobed by this system are necessarily mutually interrelated and ontologically incapable of isolation; and by this principle, the role of the "observer" in orthodox quantum mechanics is always fulfilled.

In fact, it has been suggested by Gell-Mann and Hartle that the mostly classical or "quasi-classical" behavior of the relatively highinertia macroscopic objects we typically encounter in everyday experience is, in part, a product of the decoherence effect; but it is also a product of the extremely high degree of historical correlation among facts-that is, reproduction from fact to fact along an historical route-typical of high-inertia systems, such that it is always potentia with probability valuations extremely close to one that are reproduced with great regularity along a given historical route of facts. This tendency toward potentia regulated by reproduction is typical of the high-inertia systems that constitute most of our everyday world. Schrödinger's cat, for example, is a sufficiently large enough system of facts that its own "internal environment"-apart from the unavoidable coupling with the environment "external" to the cat (the air it breathes interacting with the box it is in, interacting with the world outside the box, etc.) —easily provides the requisite degrees of freedom to produce the necessary cancellations of interfering potential states. Those potential states that survive the 
cancellations are highly regulated by closely correlated reproductions among facts constituting the historical route of the cat. The result is that if the cat is dead at time $t$, there is a probability extremely close to one that it will be dead at time $t+1$ as well. These two factors combined-decoherence, and highly regulated reproduction among facts comprising high-inertia systems-offer a clear ontological account as to why we never see live-dead cat superpositions, or any other macroscopic superpositions even though such superpositions are a necessary component of quantum mechanics. Bluntly stated by Gell-Mann: "Reams of paper have been wasted on the supposedly weird quantum-mechanical state of the cat, both dead and alive at the same time. No real quasiclassical object can exhibit such behavior because interaction with the rest of the universe will lead to decoherence of the alternatives." 17

The second benefit of this conception of system states as histories of actualities/events/facts is that it allows one to intuitively see how the so-called paradox of quantum nonlocality - arguably the most formidable obstacle to a strictly classical accommodation of quantum mechanics-is really no paradox at all. Quantum nonlocality is simply the correlation among facts in a composite quantum system, such as our example system

$$
|\Psi\rangle=\alpha\left|u_{\text {green }}\right\rangle\left|d_{\downarrow}\right\rangle\left|E_{\downarrow}\right\rangle+\beta\left|u_{\text {red }}\right\rangle\left|d_{\uparrow}\right\rangle\left|E_{\uparrow}\right\rangle
$$

when $S, D$, and $E$ entail actualities that are spatially well separated. For according to the formalism above, facts belonging to $E$, even though they may pertain to a part of the universe far removed from $S$ and $D$, nevertheless affect the actualization of potential facts belonging to $S$ and $D$.

The problem of quantum nonlocality was first formally addressed in the famous paper of Einstein, Podolsky, and Rosen (EPR) in 1935, and in 1969 an experiment was designed by Abner Shimony, John Clauser, M. Horne, and R. Holt to test the EPR argument and its newer formulation by David Bohm-that supposedly nonlocal correlations are merely statistical artifacts that derive from hidden variables, and that if these variables were disclosed, quantum mechanics would be a complete, fully deterministic theory. In 1964, John Bell demonstrated mathematically that the nonlocal correlations predicted by quantum mechanics exceeded those predicted by classical, statistical mechanics; ${ }^{18}$ his theorem was tested experimentally in 1972 by Clauser and S. Freedman, ${ }^{19}$ and in 1982 another version 
of the experiment was performed by A. Aspect, J. Dalibard, and G. Roger. ${ }^{20}$ The results of these experiments vindicated quantum mechanics by their demonstration of nonlocal interrelations in excess of those allowed for by classical statistical mechanics-to the surprise of many. In the years since these experiments, a great deal has been written about this supposedly bizarre, counterintuitive, and conceptually problematic nonlocality feature of quantum mechanics.

The interpretive strategy represented by the arguments of Einstein, Podolsky, Rosen, and Bohm entails the characterization of quantum nonlocality-as well as indefiniteness, chance, entanglement, probability - as epistemic artifacts of the quantum theory's inability to account for a multiplicity of "hidden variables" that, if disclosed, would complete the theory. Thus, quantum mechanics would be cleansed of all uncertainty so that its true essence as a fully determinate, classical theory would be revealed. Since human limitations prevent the specification of these deterministic hidden variables, however, the incompleteness of the theory with its unmovable veil of indeterminism relegates us to a statistical approximation of them. Thus the matrix of probable outcome states yielded by quantum mechanics is to be interpreted, as advocated early on by Max Born, as statistical probabilities of purely epistemic significance $^{21}$ - probabilities that describe the statistical frequency at which an experimenter measuring the position of an electron, for example, finds it in a given region around an atomic nucleus after repeated experiments. It is, in other words, the probability that one will find that the pre-established facts will fit a probable form a certain percentage of the time when a given experiment is repeated sufficiently.

This statistical interpretation of quantum mechanics is thus able to account for indefiniteness, change, probability, and entanglement by rendering these concepts merely epistemically significant, as opposed to ontologically significant. But the statistical or "hidden variables" interpretation of quantum mechanics is not so easily able to account for nonlocality; for even if nonlocal correlations between spatially well separated systems are interpreted as statistical artifacts owing to hidden variables - which, for example, might pertain to the stochastic fluctuations of the field of atomic and subatomic particles linking the two systems - the classical interactions among these particles are still limited by the speed-of-light boundary of special rela- 
tivity. Any causally significant transference of energy from one system to the other along the field-whether statistically veiled by its (apparently) stochastic fluctuations, or whether explicitly apparent as in a communication signal beamed from one system to the other-cannot exceed the speed of light. But the experiments of Aspect et al. reveal that if nonlocality is to be explained according to this classical deterministic model, these propagations along the field must be superluminal.

In answer to this difficulty, many advocates of the experimentally disconfirmed classical, "local hidden variables" interpretation moved to embrace a "nonlocal hidden variables" interpretation, which is otherwise the same in its classical interpretation of quantum mechanics-except that it allows for nonlocal interactions via a superluminal propagation of energy, in violation of special relativity. The nonlocal hidden variables theory of David Bohm, introduced in 1952,22 is the most complete and systematized incarnation of this type of theory; in it, Bohm suggests that the entire universe is permeated by an ether of point particles constitutive of an "implicate order" that can be described and accounted for via quantum mechanics. This not only allows for nonlocal interactions via superluminally propagated energy, characterized as a kind of "shock wave," similar to the "pilot wave" model proposed by de Broglie in 1927; it also renders the universe fundamentally time-reversal invariantthat is, fundamentally deterministic, such that past, present, and future are all mutually internally related.

To illustrate this fundamental deterministic symmetry of Bohm's "implicate order," I refer again to the experimental device described by Bohm and his colleague B. J. Hiley referenced in chapter 1:

This device consists of two concentric glass cylinders; the outer cylinder is fixed, while the inner one is made to rotate slowly about its axis. In between the cylinders there is a viscous fluid, such as glycerine, and into this fluid is inserted a droplet of insoluble ink. Let us now consider what happens to a small element of fluid as its inner radius moves faster than its outer radius. This element is slowly drawn out into a finer and finer thread. If there is ink in this element it will move with the fluid and will be drawn out together with it. What actually happens is that eventually the thread becomes so fine that the ink becomes invisible. However, if the inner cylinder is turned in the reverse direction, the parts of this thread will retrace their steps. (Because the viscosity is so high, diffusion can be neglected.) Eventually 
the whole thread comes together to reform the ink droplet and the latter suddenly emerges into view. If we continue to turn the cylinder in the same direction, it will be drawn out and become invisible once again.

When the ink droplet is drawn out, one is able to see no visible order in the fluid. Yet evidently there must be some order there since an arbitrary distribution of ink particles would not come back to a droplet. One can say that in some sense the ink droplet has been enfolded into the glycerine, from which it unfolds when the movement of the cylinder is reversed.

Of course if one were to analyse the movements of the ink particles in full detail, one would always see them following trajectories and therefore one could say that fundamentally the movement is described in an explicate order. Nevertheless within the context under discussion in which our perception does not follow the particles, we may say this device gives us an illustrative example of the implicate order. And from this we may be able to obtain some insight into how this order could be defined and developed. ${ }^{23}$

As regards the phenomenon of quantum nonlocality, Bohm and Hiley suggest a modification of this model:

Suppose that we consider two particles initially at different positions, one represented by red ink and the other by blue ink. As we enfold these into the fluid, red ink particles and blue ink particles will eventually intermingle, so that in a given region we could not say that the ink droplets were really separate. Nevertheless when the cylinder was turned back, red and blue ink particles would begin to separate to form respectively red and blue droplets. It is clear that the ground of the particular behaviour of the visible droplets is global and that disturbances in this ground can lead to correlated changes in this behaviour.

In our model the appearance in perception of what seems to be a pair of separate ink droplets can therefore be deceiving. Actually these apparently separate ink droplets are internally related to each other. On the other hand, as conceived in the explicate order, such droplets would only be externally related. This is one of the most important new features of the implicate order. What may be suggested is that this feature may give further insight into the meaning of quantum properties such as mutual participation and nonlocality. ${ }^{24}$

Again, the mechanism by which the implicate order is mediated between the nonlocal actualities is a "pilot wave" propagated throughout the ether of hidden point particles constituting the im- 
plicate order. But this method of mediation is not compatible with special relativity because it is not Lorentz invariant. Bohm's interpretation requires, in other words, that spatially well separated systems be connected instantaneously (and therefore superluminally) by the pilot wave. The deterministic implications of this requirement were explored in chapter 1 , as was the related implication of fundamental temporal symmetry. With respect to the concept of instantaneous nonlocal interactions, however, these implications become intimately bound up in the possibility of faster-than-light communication. Given the pilot wave's instantaneous connection of spatially well separated systems in Bohm's theory, in violation of special relativity, superluminal communication should be possible; that it has not yet been achieved is, according to Bohm, primarily a consequence of experimental limitations. He writes:

Of course, until we are able to carry out experiments on individual processes that are more accurate than the limits set by the uncertainty principle, it will not be possible to use quantum nonlocality for the purpose of sending signals. . . Any attempt to send a signal by influencing one of a pair of particles under EPR correlation will encounter the difficulties arising from the irreducibly participatory nature of all quantum processes. If for example we tried to 'modulate' the overall wave function so that it could carry a signal in a way similar to what is done by a radio wave, we would find that the whole pattern of this wave would be so fragile that its order could change radically in a chaotic and complex way. As a result no signal could be carried. ${ }^{25}$

The ether of point particles, in other words, is stable enough to produce EPR-like nonlocal correlations superluminally, but not stable enough to produce a communicative signal superluminally. This difficulty aside, the central point here is that in Bohm's hidden variables theory, the experimentally verifiable phenomenon of quantum nonlocality is accounted for by a dynamical mechanism which is fundamentally incompatible with special relativity. When one recalls that the original point of the hidden variables interpretations of quantum mechanics was to render it a classically deterministic theory, the instantaneous nonlocal connection of particles by a Lorentz noninvariant, superluminal pilot wave seems a serious price to paythe salvaging of fundamental classicality by means of selective dispensations from special relativity, one of its most central principles.

By contrast, quantum nonlocality, when interpreted via a satisfactory ontological framework such as that proposed by Żurek, Omnès, 
Gell-Mann, et al., is entirely reasonable and classically intuitive-so much so, in fact, that one can use "real world" situations to demonstrate this interpretation of quantum nonlocality (a good indicator of the coherence of this ontological framework). The following quantum nonlocality analogy is an example of what is sometimes referred to as a "Cambridge change" - a term that likely derives from P. T. Geach's use of it in his 1969 book God and the Soul. ${ }^{26}$ The term refers to Cambridge philosophers such as Bertrand Russell and J. M. E. McTaggart, who had considered the idea of changing descriptive qualifications (e.g., per this discussion, "changing quantum mechanical states") as ontologically significant to the object of the description. Geach writes:

The only sharp criterion for a thing's having changed is what we may call the Cambridge criterion (since it keeps on occurring in Cambridge philosophers of the great days, like Russell and McTaggart): The thing called " $x$ " has changed if we have " $\mathrm{F}(x)$ at time $t$ " true and " $F(x)$ at time $t$ " false, for some interpretation of "F", " $t$ ", and " $t$ ". But this account is intuitively quite unsatisfactory. By this account, Socrates would after all change by coming to be shorter than Theaetetus; moreover, Socrates would change posthumously (even if he had no immortal soul) every time a fresh schoolboy came to admire him; and numbers would undergo change whenever e.g. five ceased to be the number of somebody's children.

The changes I have mentioned, we wish to protest, are not "real" changes; and Socrates, if he has perished, and numbers in any case, cannot undergo "real" changes. I cannot dismiss from my mind the feeling that there is a difference here; and I suggest that when we have a narrative proposition corresponding to a "real" change, there is individual actuality — an imperfect actuality, Aristotle calls it - that is the change; but not, when a mere "Cambridge" change is reported. (Of course there is a "Cambridge" change whenever there is a "real" change; but the converse is not true.) But it would be quite another thing to offer a criterion for selecting, from among propositions that report at least "Cambridge" change, those that also report "real" change (given they are true); and I have no idea how I could do that. ${ }^{27}$

The decoherence or "consistent histories" interpretations of quantum mechanics provide an excellent solution to the question of such Cambridge changes, even those that entail nonlocal correlations. The key, as we have seen, lies in two fundamental principles: First, the concept of ontologically significant potentia, and the un- 
derstanding that actualities (what Geach refers to as "real" changes) and potentia constitute the two fundamental species of reality; and second, the fundamental characterization of objects as serial, historical routes of quantum events-quantum actualizations. Thus, as the potentia associated with an historical route of quantum mechanical state specifications change, then in some sense the system described by this historical route must also change. The potentia associated with a history, in other words, contribute to its definition in an ontologically significant way, just as the actualities associated with a history do; the difference is that the potentia associated with a quantum mechanical history can be affected nonlocally by correlation with the actualities of other histories. All that is required is the inclusion of the correlated histories in some broader, shared environmental history by which logically necessary consistency conditions become operative. These consistency conditions are ultimately grounded in the logical principles of noncontradiction and the excluded middle, the foundation of any and all logical constructions, including, obviously, the mathematics that lie at the heart of quantum theory.

Consider, for example, the history of actualities/events/facts comprised by a traveling salesman, whose pregnant wife in California is soon to deliver. Certain of the actualities/events/facts belonging to the salesman pertain to his location, which is somewhere in Hong Kong, far away from his wife. Nevertheless, the history describing the salesman is correlated with the history describing his wife, such that as soon as she gives birth, we can say that the salesman's history has been augmented in a definite, objective way, evinced by asking the simple question: When does the salesman become a father? When, in other words, are the potentia objectively pertinent to his future affected? According to a classical mechanical interpretation of this situation, the answer would be: As soon as his wife is able to communicate this fact to him-communication limited to the speed of light, according to special relativity.

But according to a quantum mechanical interpretation of this situation, the history of states describing the salesman instantly changes as soon as his wife gives birth, by virtue of the fact that his state history is correlated with that of his wife. The correlations of their two histories are accounted for by two concepts: (i) Both histories are correlated with an environmental history, which is itself englobed by the universal history $|\Psi\rangle$, and therefore all these correlated 
histories must be mutually consistent; (ii) the consistency among the salesman and wife histories is a function of the decoherence effect, which eliminates any incompatible, interfering histories in the pure state density matrix, such that the alternative histories comprising the reduced state density matrix are consistent, mutually exclusive and exhaustive probabilities.

The fact that the history of states describing the salesman changes as soon as his wife gives birth does not, as has been suggested in some discussions of the real experiments involving quantum nonlocality, imply any "superluminal communication" in violation of special relativity. Theoretical exploration of the possibility of such faster-than-light communication via quantum nonlocality has revealed that correlations among nonlocal histories cannot involve the communication of information. Put another way, quantum mechanics allows the actualities of one system to causally affect the potentia of a spatially well separated system by virtue of the correlated histories of the two systems; quantum mechanics does not, however, allow spatially well separated systems to influence one another causally. This distinction between "causal affection of potentia by logically prior actuality" and "causal influence of actualization by temporally prior actuality" is perhaps negligible in classical mechanics, where both constitute the qualification of material substance by quality. In an ontological interpretation of quantum mechanics, however, the distinction between causal affection of potentia and causal influence of actualization is directly related to and necessitated by the ontological significance of potentiality. For as the evolution of a state or history of a system is driven by the potentia associated with the system-potentia that are subsequently and consequently related to all logically prior facts in the closed system of the universe-an affection upon these potentia does not necessarily entail causal influence upon the quantum mechanical actualization of the system.

The state history of the salesman, in other words, involves not only the actualities/events/facts subsumed by his state history as it exists, but also the potential actualities/events/facts subsumed by his state history as it evolves; and it is these potentia that are affected nonlocally. Though he is not aware of his state change, for the same reason superluminal communication is not possible in quantum mechanics, the potentia associated with the salesman's subsequent and consequent history - and the role of these potentia in forming the probabilities for their actualization-are affected, and in an ontolog- 
ically significant way. One sees here the rehabilitation of the Aristotelian intuition that a thing is not only defined by what it was in the past, but also by what it might become in the future-an intuition we typically restrict to those occasions wherein we define ourselves. The decoherent histories interpretations of quantum mechanics, however-interpretations which stress that quantum mechanics predicts probable potential outcome states and not determined actual outcome states-reveal that it is an intuition which we might sensibly apply to the whole of nature.

The intuitive ontological concepts discussed in these real-world examples of nonlocality are identical to those used in interpreting quantum nonlocality in the famous EPR-type laboratory experiments performed by Aspect et al., which is but one indicator of the coherence of the ontological interpretation of quantum mechanics presented thus far.

The third benefit of this interpretation, closely related to nonlocality, is that it mediates the controversy in physics, originating in Boltzmann's kinetic theory, between (i) the temporal symmetry implied by the time-reversal invariance of classical dynamics and (ii) the temporal asymmetry - the one-way directionality of timeexemplified by the second law of thermodynamics. Since it is fundamentally the time-independent form of the Schrödinger equation that describes the evolution of the state of a system (or a history of such states) from an actual state to an integration of potential states, this evolution essentially occurs "out of time." It is the actualization of one of these potential states - the discontinuous punctuation of this symmetrically atemporal continuum of potentia-which results in an asymmetrical temporality-the only temporality we know. By this ontological interpretation of quantum mechanics, then, asymmetrical time is in fact a product of the actualizations of potentia, rather than a background against which such actualizations occur. ${ }^{28}$ One manifestation of this is the question: "What happens to an electron in between observations?" Heisenberg answers that quantum mechanics "does not allow a description of what happens between two observations. Any attempt to find such a description would lead to contradictions; this must mean that the term "happens' is restricted to observation." 29 Asking, "What happens to an electron in between observations?" is, by the decoherence-based interpretations of quantum mechanics, the same as asking, "What happens to an electron in between actualizations?" The answer, of 
course, is that nothing "happens" to it (apart from its own internal quantum mechanical state evolution) since the electron does not exist as an actual entity in between actualizations; it exists only as a potential entity in between actualizations. The only electrons that are "actual" are those of the past relative to an evolving actualization in the present. The past is thus settled and the future remains open, with subsequent actualizations causally influenced by the objective data of past actualities, but not determined by these data.

Temporal asymmetry is crucial to an ontological interpretation of quantum mechanics, since logically ordered causal dynamics are impossible apart from such asymmetry; but temporal asymmetry is neither provided nor accounted for by the Schrödinger equation in either its time-independent or time-dependent forms; it is a product, rather, of the actualization of potentia, and therefore beyond the scope of the quantum formalism. Yet temporal and logical asymmetry must be a part of any coherent ontological interpretation of quantum mechanics. Heisenberg writes: "The transition from the 'possible' to the 'actual' is absolutely necessary here and cannot be omitted from the interpretation of quantum theory. At this point, quantum theory is intrinsically connected with thermodynamics in so far as every act of observation is by its very nature an irreversible process; it is only through such irreversible processes that the formalism of quantum theory can be consistently connected with actual events in space and time." 30

The long-sought-after physical mechanism responsible for the actualization of potentia - that is, the existence of facts-remains elusive, however. We have already explored the reason proposed by a classical statistical interpretation: Asymmetrical causal relations such as those pertaining to thermodynamics are in fact purely symmetrical, time-reversal-invariant relations whose symmetry lies beneath a veil of hidden variables. In these hidden variables, in other words, lies the key to unscrambling an egg. "Irreversibility," wrote Born, "is a consequence of the explicit introduction of ignorance into the fundamental laws." 31

But as already discussed, the inability of quantum mechanics to account for the actualization of potentia or the temporally asymmetrical relations that obtain from such actualizations, is not problematic given that quantum mechanics presupposes and anticipates the existence of facts; this is evinced in the concepts of state evolution, 
probability, and history. "One may consider," writes Roland Omnès, "that the inability of the quantum theory to offer an explanation, a mechanism, or a cause for actualization is in some sense a mark of its achievement. This is because it would otherwise reduce reality to bare mathematics and would correspondingly suppress the existence of time." 32

The advantage of the decoherence-based interpretations of quantum mechanics, however, is that the process of negative selection entails the necessary integration of noninterfering potentia into sets of mutually exclusive and exhaustive, probability-valuated potential outcomes. And without this process, it is unclear whether or not the actualization of potentia could occur at all. Omnès writes that though it is not the case with respect to the Schrödinger equation of the quantum formalism, the direction of time in a coherent, logical interpretation of quantum mechanics is "restricted by decoherence in such a way that the direction of time must be the same as the direction of time in thermodynamics. It should be observed that this statement is not an intrinsic necessity of pure quantum mechanics. If the universe contained only two or three particles [such that decoherence were not likely], there would be no such restriction and one would be allowed to choose arbitrarily the direction of time in logic." 33

The actualization of potentia-the existence of facts-constitutes an asymmetrizing punctuation of the continuum of potentia whose superposed, symmetrical, and therefore "logically atemporal" relations are reflected in the logically atemporal dynamics of quantum mechanical state evolution and its time-independent Schrödinger equation. Even the time-dependent form of the Schrödinger equation is "logically atemporal," since as a linear, deterministic equation, it is symmetrical with respect to time-reversal. But it must be recalled that the Schrödinger equation is not "deterministic" in the sense that it is determinative of a unique, actual outcome state; it is determinative of probability-valuated potential outcome states. Thus a time reversal transformation applied to the time-dependent Schrödinger equation does not yield a determined, unique state, but rather a superposition of potential states.

Apart from the actualization of potentia, which lies beyond the scope of the Schrödinger equation, a quantum mechanical evolution is, therefore, logically atemporal. Logical, asymmetrical temporality, 
in other words, is not the "background" against which the Schrödinger equation, in either its time-dependent or more fundamental time-independent forms, operates. Logical, asymmetrical temporality is a product of the actualization of potentia-the evolution of probability to fact; it is not a metric by which the evolution of potentia to probabilities can be measured. For without the existence of facts, there is nothing to measure temporally. Though an explanation for the existence of facts lies beyond the scope of quantum mechanics, it is both presupposed and anticipated by the mechanics; and indeed, the existence of facts is necessary if the mechanics is to account for the physical, causal interrelations characteristic of our experiences, and for the temporal asymmetry such interrelations entail. Omnès writes:

The existence of actual facts can be added to the [quantum] theory from outside as a supplementary condition issued from empirical observation. The structure of time must then be modified accordingly. Time must be split into a past, a present, and a future having very different qualities. Present and past are uniquely defined while the future must remain potential and subject to probabilistic expectations. This structure of time, so obvious from the standpoint of observation, turns out to be necessary from a theoretical standpoint. ${ }^{34}$

Temporal asymmetry guarantees the uniqueness of facts in this ontological interpretation of quantum mechanics; yet it must be stressed then that facts integrated into states and histories of states, in a sense, evolve to become new facts with the creation of each new state, in augmentation of the history to which the state applies. Past actualities are real—and in a sense, then, immortal — given that the actualization of any and every novel state of the universe, in augmentation of the history describing the universe, entails an integration of all facts belonging to the universe. These many facts are integrated, according to quantum mechanics, to form a single state; and the history to which the state belongs is thus augmented. We make use of past facts, then, via recollection, which is a novel-and, as are all actualities, unique-re-creation of the past. All past actualities are thus recorded in the actualization of every subsequent staterecorded in a system, in a measuring apparatus, or in the environment. It is temporal asymmetry that relegates us to using perpetually re-created records of past actualities rather than the actualities 
themselves; but it is also temporal asymmetry that, by this same relegation, provides for the objective immortality of every fact in the universe. Omnès writes:

Past facts are not absolutely real; they only were real. One can never indicate a past fact by pointing a finger at it and saying "that." One must call for a memory or use a record, a note, a photograph. Nevertheless, the derivation of a unique past is possible because quantum mechanics allows for the existence of memory and records. It goes without saying that everybody always takes this structure of time for granted but, up till then, it was always tacitly added to our understanding of physics and never supposed to be a consequence of it. . . What we observe in reality is always something existing right now, even if we interpret it as a trace of an event in the past, whether it be a crater on the moon, the composition of a star atmosphere, or the compared amounts of uranium and lead in a rock. ${ }^{35}$

\section{SUMMARY}

Now that we have described the quantum mechanical evolution of the state of a system-the forward, historical movement of facts from actuality to potentiality to actuality, driven by nothing other than their necessary mutual interrelations-let us formulate a final interpretation of this evolution as it applies to our idealized traffic signal system. It should first be noted that by most interpretations, quantum mechanics cannot be applied sensibly to the evolution of macroscopic systems; however, the decoherence effect, as we have seen, has served to bridge the conceptual border between "quantum reality" and "classical reality" as demarked by Bohr with his principle of complementarity. The "classicality" of macroscopic systems can now be interpreted most fundamentally as an effect of the mutual interrelations among the facts comprised by such systems. This is not to suggest that macroscopic systems are best described quantum mechanically; only that they are most fundamentally described by quantum mechanics.

Returning now to the interpretation of the evolution of our redgreen traffic-signal system, let us define all the systems involved:

Universe

The system of all facts/actualities. We shall represent the state of this system-the maximal specification of all facts belonging to it-with 
the state vector $|\Psi\rangle$. It is understood that individual states-that is, "snapshots" of any system-and serial histories of states, are mechanically equivalent, and for the purposes of this discussion are interchangeable and will both be represented by the same state vector.

\section{Measured System}

The system of all facts/actualities belonging to the traffic signal, whose state shall be represented by the state vectors $\left|u_{\text {green }}\right\rangle$ or $\left|u_{\text {red }}\right\rangle$. It is understood that the nomenclature used for the state vectors $\left|u_{\text {green }}\right\rangle$ or $\left|u_{\text {red }}\right\rangle$ indicate that these states only specify the facts pertaining to the status of the red and green lights; all other facts belonging to the Measured System are ignored.

\section{Detector}

The system of all facts/actualities belonging to the detector-an up/ down pointer apparatus, whose state shall be represented by the state vectors $\left|d_{\downarrow}\right\rangle$ and $\left|d_{\uparrow}\right\rangle$. The detector is constructed so that when sufficiently interacted with the traffic-signal system, state $\left|d_{\downarrow}\right\rangle$ correlates with state $\left|u_{\text {green }}\right\rangle$, and state $\left|d_{\uparrow}\right\rangle$ correlates with state $\left|u_{\text {red }}\right\rangle$. All facts belonging to the Detector that do not pertain to the direction of the pointer are ignored.

\section{Environment}

The system of all facts/actualities other than those represented by $\left|u_{\text {green }}\right\rangle,\left|u_{\text {red }}\right\rangle,\left|d_{\downarrow}\right\rangle$ and $\left|d_{\uparrow}\right\rangle$. These facts may thus belong to the environment external to the Measured System and the Detector, or they may belong to the environment internal to them, comprising all facts not specified by $\left|u_{\text {green }}\right\rangle,\left|u_{\text {red }}\right\rangle,\left|d_{\downarrow}\right\rangle$, and $\left|d_{\uparrow}\right\rangle$ —that is, facts that have been ignored. The state of the Environment is represented by the state vector $\left|E_{0}\right\rangle$.

It is understood that the facts belonging to the Measured System, Detector, and Environment also belong to $|\Psi\rangle$, forming "subsystems" of $|\Psi\rangle$, and their grouping into subsystems in no way vitiates the mutual and necessary interrelations with all facts belonging to $|\Psi\rangle$. Hence, the states of the Measured System, the Detector, and the Environment-as well as the histories of these states-are all objectively mutually correlated. An analogous example of such correlation can be seen when considering the local histories describing California and Maine, respectively, within the larger history of the 
United States; facts belonging to each subhistory also belong to a larger history englobing both, which ensures an objective correlation among the facts specified by these histories. Similarly, one cannot exist in Paris and New York simultaneously, for the superposition of mutually interfering histories such a feat would require are eliminated via the decoherence effect.

The measurement of our traffic-signal system, then, most fundamentally entails a specification of a subset of facts belonging to the universe, relative to a single fact or subset of facts, referred to here as "the indexical eventuality" belonging to the subset of facts referred to as "the Detector." In an ontological interpretation of quantum mechanics, every fact must be understood as capable of fulfilling the role of "indexical eventuality" or "Detector" during the evolution of $|\Psi\rangle$, which, according to some interpretations, locks quantum mechanics into a fog of sheer subjectivity. But this condemnation overlooks the ontological requirement of the correlation among actualities (and histories of actualities) as discussed in the preceding paragraph, without which quantum mechanics-and indeed the world itself-would cease to be accessible to reason. Quantum mechanics is not, therefore, rooted in subjectivity, but rather merely in relativity; quantum mechanics does not entail the subjective relations among facts-merely the relations among facts relative to a given fact or subset of facts. Since the formalism requires that we follow only one indexical eventuality at a time, we shall select the pointer needle of our Detector, which was intended for the purpose.

We begin, then, with the mutually correlated states describing the System, the Detector, and the Environment-i.e., the Universewhose state is described by $|\Psi\rangle$ and reflects the state of these systems relative to a single measurement interaction:

$$
|\Psi\rangle=\alpha\left|u_{\text {green }}\right\rangle\left|d_{\downarrow}\right\rangle\left|E_{\downarrow}\right\rangle+\beta\left|u_{\text {red }}\right\rangle\left|d_{\uparrow}\right\rangle\left|E_{\uparrow}\right\rangle
$$

This expression simply groups all possible projections of $|\Psi\rangle$ into two subspaces, $\mathscr{E}$ and $\mathscr{E}^{\perp}$, determined by the pointer needle of the Detector system, our choice of indexical eventuality. If subspace $\mathscr{E} \downarrow$ consists of all vectors representing potential states (or potential histories) where the pointer is down $\left|d_{\downarrow}\right\rangle$, then subspace $\mathscr{E}_{\downarrow}^{\perp}$ consists of all vectors representing potential states (or potential histories) where the pointer is not down. ( $E_{\uparrow}$, for example, would belong to 
this subspace.) The manifold possible projections of $|\Psi\rangle$ upon $\mathscr{E}$ are integrated into the term

$$
\alpha\left|u_{\text {green }}\right\rangle\left|d_{\downarrow}\right\rangle\left|E_{\downarrow}\right\rangle
$$

interpreted as: "All potential states of the universe where the pointer is down and the light is green, with all other actualities unspecified." Every potential state or history of states integrated in $\alpha\left|u_{\text {green }}\right\rangle\left|d_{\downarrow}\right\rangle\left|E_{\downarrow}\right\rangle$ thus tells some unique, potential story about every actuality in the universe, all of which agree upon the specification of the color of the traffic signal and the direction of the pointer needle. The complex coefficient $\alpha$ represents the length of this integrated projection and will ultimately represent the probability that the traffic signal will be green and the pointer needle of the detector will point downwardthat is, the quantified valuation of this potential fact.

Likewise, the possible projections of $|\Psi\rangle$ upon $\mathscr{E}_{\uparrow}$ are integrated into the term $\beta\left|u_{\text {red }}\right\rangle\left|d_{\uparrow}\right\rangle\left|E_{\uparrow}\right\rangle$, with the complex coefficient $\beta$ representing the length of this integrated projection, and ultimately the probability that the traffic signal will be red and the pointer needle of the detector will point upward.

However,

$$
|\Psi\rangle=\alpha\left|u_{\text {green }}\right\rangle\left|d_{\downarrow}\right\rangle\left|E_{\downarrow}\right\rangle+\beta\left|u_{\text {red }}\right\rangle\left|d_{\uparrow}\right\rangle\left|E_{\uparrow}\right\rangle
$$

also includes nonsensical, mutually interfering superpositions of states whose vectors belong to neither $\mathscr{E}_{\downarrow}$ nor $\mathscr{E}_{\uparrow}$ exclusively. These are revealed in the correlated, pure state density matrix of potential states of $|\Psi\rangle$ :

$$
\begin{aligned}
\rho^{c} & =|\Psi\rangle\langle\Psi| \\
& =|\alpha|^{2}\left|u_{\text {green }}\right\rangle\left\langle u_{\text {green }}|| d_{\downarrow}\right\rangle\left\langle d_{\downarrow} \| E_{\downarrow}\right\rangle\left\langle E_{\downarrow}\right| \\
& +\boldsymbol{\alpha} \boldsymbol{\beta}^{*}\left|\boldsymbol{u}_{\text {green }}\right\rangle\left\langle\boldsymbol{u}_{\text {red }}|| \boldsymbol{d}_{\downarrow}\right\rangle\left\langle\boldsymbol{d}_{\uparrow} \| \boldsymbol{E}_{\downarrow}\right\rangle\left\langle\boldsymbol{E}_{\uparrow}\right| \\
& +\boldsymbol{\alpha}^{*} \boldsymbol{\beta}\left|\boldsymbol{u}_{\text {red }}\right\rangle\left\langle\boldsymbol{u}_{\text {green }}|| \boldsymbol{d}_{\uparrow}\right\rangle\left\langle\boldsymbol{d}_{\downarrow}\right\rangle\left\langle\boldsymbol{E}_{\downarrow}\right|\left\langle\boldsymbol{E}_{\downarrow}\right. \\
& +|\boldsymbol{\beta}|^{2}\left|u_{\text {red }}\right\rangle\left\langle u_{\text {red }}|| d_{\uparrow}\right\rangle\left\langle d_{\uparrow} \| E_{\uparrow}\right\rangle\left\langle E_{\uparrow}\right|
\end{aligned}
$$

where the off-diagonal terms in boldface represent superpositions of interfering and thus nonsensical states incapable of integration.

The correlation between our System and Detector and the Environment produces such a vast multiplicity of potential states, each represented by a unique projection in $\mathscr{E}_{\downarrow}$ or $\mathscr{E}_{\uparrow}$, that the majority of these vectors cancel each other out, thereby eliminating the interfer- 
ing superpositions represented by the off-diagonal terms in boldface. This cancellation is represented mathematically by a "trace-over" or "sum-over" of the unmeasured degrees of freedom belonging to the Environment. Eliminating these unmeasured, potential environmental facts, in essence, eliminates the superpositions of incompatible, potential universal states associated with them. The result of this process of negative selection is the decoherence of the potential outcome states, such that those that remain are mutually exclusive and exhaustive potentia (in satisfaction of the logical principles of noncontradiction and the excluded middle), each valuated as a probability. These are represented by a reduced density matrix:

$$
\rho^{r}=|\alpha|^{2}\left|u_{\text {green }}\right\rangle\left\langle u_{\text {green }}|| d_{\downarrow}\right\rangle\left\langle\left. d_{\downarrow}|+| \beta\right|^{2} \mid u_{\text {red }}\right\rangle\left\langle u_{\text {red }}|| d_{\uparrow}\right\rangle\left\langle d_{\uparrow}\right|
$$

where $|\alpha|^{2}+|\beta|^{2}=1$, such that each term represents a potential state or history of states valuated as a probability.

We can now briefly summarize the ontological interpretation of the quantum mechanical interaction described above as follows:

1. The specification of the state of a system of facts necessarily entails the state of the universe which subsumes the system. Further, the specification of the state of a system is itself a fact-a novel fact, born of the process by which the state is specified. Thus, the specification of the state of a system ultimately entails the specification of an historical route of states, newly augmented with each new specification. Every specification of a system state, then, entails the evolution of a state from a multiplicity of actualities, through integrations of potentialities, to a novel actuality.

2. The specification of the state of a system of facts begins with the integration of all facts relative to a particular fact referred to as the "indexical eventuality." The relativity of this integration is both objective and subjective. It is objective in that it is an integration of facts, and it is subjective in that the uniqueness of the indexical eventuality conditions the particular form of the integration. The latter is reflected in quantum mechanics by the fact that the preferred basis is determined by the indexical eventuality.

3. There are manifold potential integrations of potential facts relative to any particular indexical eventuality, and each potential integration amounts to a potential state (or potential historical route of states) of the system. Since each indexical eventuality is unique, each produces its own particular forms of these integrations, such that for 
each indexical eventuality, there exist potential facts which are incapable of integration in a single potential state. If, for example, there are two potential facts pertaining to a particle's position and these potential facts disagree, they cannot be integrated together in a "consistent" potential state or history of states. In quantum mechanics, these mutually interfering potential facts or potential states are bound into coherent superpositions, represented in the formalism by the off-diagonal terms in the pure state density matrix.

4. A process of negative selection is therefore required to remove these mutually interfering potentia which are incapable of integration. This process is possible only by virtue of the integration of all facts, and not only those belonging to the measured system; for it is the correlation of facts belonging to this system with unmeasured and relatively irrelevant facts environmental to it that produces a sufficient number of potential states that (i) particular forms of integration with compatibilities and incompatibilities are possible at all and (ii) there exist a sufficient number of incompatible potential states that their propensities for actualization are mutually cancelled. The elimination, by negative selection, of superpositions of interfering, incompatible potentia is achieved by ignoring all potential facts that are essentially unrelated to the pointer basis of the indexical eventuality (the "unmeasured degrees of freedom" of the environment). In other words, the elimination of this irrelevant multiplicity of detail of environmental potential facts effectively entails the elimination of any interfering, coherent superpositions of potential states associated with them. The result is a "decoherent" set of noninterfering potential states, represented in the formalism by the reduced density matrix, that are (i) logical, in that they are mutually exclusive and exhaustive, in satisfaction of the principles of noncontradiction and the excluded middle, and (ii) have propensities for actualization that have become valuated as probabilities.

5. This entire process is predicated upon the a priori existence of facts, as implied, for example, in the following three concepts: (i) state evolution (evolution from antecedent fact, through potentia, to novel fact); (ii) histories of states (histories being serial routes of facts perpetually augmented by novel facts); and (iii) probability (probability that an antecedent fact will become a particular potential novel fact according to a quantifiable valuation). It is the probability valuation of the mutually exclusive and exhaustive potential 
states that governs, and therefore guarantees, the actualization of a unique outcome state. The entire process, therefore, occurs relative to both $(i)$ the indexical eventuality as an actual subject, participant in the system whose state is being specified, and (ii) the indexical eventuality as the actual satisfaction of the valuations terminal of that part of the process described by quantum mechanics. For as probabilities, these valuations presuppose and anticipate this satisfaction. It is the valuation of potentia, then, and the process productive of this valuation-rather than the actualization of potentia-that quantum mechanics describes.

\section{Notes}

1. Werner Heisenberg, Physics and Philosophy (New York: Harper Torchbooks, 1958), 185.

2. Murray Gell-Mann, The Quark and the Jaguar: Adventures in the Simple and the Complex (New York: W. H. Freeman, 1994), 167.

3. Heisenberg, Physics and Philosophy, 143.

4. Ibid., 55.

5. Ibid., 53.

6. Wojciech Żurek, "Decoherence and the Transition from the Quantum to the Classical." Physics Today 44, no. 10 (1991): 36-44.

7. Wojciech Żurek, "Letters," Physics Today 46, no. 4 (1993): 84.

8. John von Neumann, Mathematical Foundations of Quantum Mechanics (Princeton, N.J.: Princeton University Press, 1955).

9. Żurek, "Decoherence and the Transition from the Quantum to the Classical," 39.

10. Ibid., 41.

11. Ibid., 40 .

12. Robert J. Griffiths, "Consistent Histories and the Interpretation of Quantum Mechanics," Stat Phys. 36 (1984): 219; see also Griffiths, Consistent Quantum Theory (Cambridge: Cambridge University Press, 2002).

13. Roland Omnès, The Interpretation of Quantum Mechanics (Princeton, N.J.: Princeton University Press, 1994).

14. Murray Gell-Mann and James Hartle, "Strong Decoherence," in Proceedings of the Fourth Drexel Symposium on Quantum Non-integrabilityThe Quantum-Classical Correspondence, Drexel University, September 1994.

15. The "discrete histories" interpretation advocated by Griffiths, GellMann, Hartle, and Omnès entails serially ordered sequences of quantum 
mechanical states-that is, time sequences of projection operators. This idea is related to an earlier conception of continuous quantum mechanical histories by Richard Feynman. But Feynman's histories ("Feynman paths") are "continuous histories" rather than "discrete," decoherent histories.

16. Gell-Mann and Hartle, "Strong Decoherence."

17. Gell-Mann, The Quark and the Jaguar, 153.

18. John Bell, "On the Einstein Podolsky Rosen Paradox," Physics 1, no. 3 (1964): 195-200.

19. S. Freedman and J. Clauser, "Experimental Test of Local Hidden Variables Theories," Phys. Rev. Lett. 28 (1972): 934-941.

20. A. Aspect, J. Dalibard, and G. Roger, "Experimental Test of Bell's Inequalities Using Time-Varying Analyzers," Phys. Rev. Lett. 44 (1982): 1804-1807.

21. Max Born, "Bemerkungen zur statistischen Deutung der Quantenmechanik" in Werner Heisenberg und die Physik unserer Zeit, ed. F. Bopp (Braunschweig: F. Vieweg, 1961), 103-118.

22. David Bohm, "A Suggested Interpretation of the Quantum Theory in Terms of 'Hidden' Variables," Phys. Rev. 85 (1952): 166, 180. See also David Bohm and B. J. Hiley, The Undivided Universe: An Ontological Interpretation of Quantum Theory (London: Routledge, 1993).

23. David Bohm and B. J. Hiley, The Undivided Universe: An Ontological Interpretation of Quantum Theory (London: Routledge, 1993), 358.

24. Ibid., 359.

25. Ibid., 283-284.

26. P. T. Geach, God and the Soul (London: Routledge, 1969).

27. Ibid., 71-72.

28. It is possible, in fact, to relate this temporal symmetry-asymmetry to the spatial symmetry-asymmetry of quantum nonlocality discussed previously. Where classical dynamics requires the symmetrical, mutual independence of spatially well separated systems-that is, that they be symmetrically externally related only-quantum dynamics allows for nonlocal, asymmetrical interrelations between spatially well separated systems. Thus, an actualization of a potential state in one system, as we have seen, affects the potential states in the other system, thereby affecting the potential subsequent actualizations of this system.

29. Heisenberg, Physics and Philosophy, 52.

30. Ibid., 137-138.

31. Max Born, Natural Philosophy of Cause and Chance (Oxford: Oxford University Press, 1949), 72.

32. Omnès, The Interpretation of Quantum Mechanics, 494.

33. Ibid., 318.

34. Ibid., 508.

35. Ibid., 344. 